T. Higa

Nagoya Math. J.

Vol. 106 (1987), 49-77

\title{
ON THE EQUIVALENCE PROBLEM AND INTEGRATION OF DIFFERENTIAL SYSTEMS
}

\author{
TATSUO HIGA
}

\section{Introduction}

The purpose of the presnet paper is to study the relationship between the theory of Lie pseudogroups and the problem of integration of differential systems (cf. [6] pp. 30-47).

Let (S) be a Lie pseudogroup on a manifold $M$ and $S$ a differential system on $M$. Let $\&(S)$ denote the largest subpseudogroup of \&S leaving $S$ invariant. Then the problems to be considered may be stated as follows.

A) Classify differential systems on $M$ under the action of (s).

B) For each differential system $S$ on $M$, determine the structure of (S) $(S)$.

C) Using the structure of $\$(S)$, reduce the problem of integration of $S$ to that of some auxiliary differential systems, each of which is invariant under the action of a Lie pseudogroup and irreducible in a sense.

To study these problems, we use the theory of Lie pseudogroups which is developed in [7]. The problems A) and B) are subordinate to the socalled general equivalence problem (see [2] $\S \S 11-13$ ). The problem $\mathrm{C}$ ) is motivated by the classical scheme of $\mathrm{S}$. Lie for the problem of integration (see [8] and [9] Introduction).

In Section 1, we recall briefly the theory of Lie pseudogroups. A Cartan system is a pair $(P, C)$ consisting of a manifold $P$ and an "invariant system" $C$ on $P$. We can define an effective action of $(P, C)$ on a manifold $M$. Then the action yields a Lie pseudogroup \&s on $M$. $(P, C)$ is called a defining Cartan system of \&s.

In Section 2, we shall study the equivalence problem of Pfaffian (differential) systems. Let $(P, C)$ and $\&$ be as above. For each Pfaffian system $S$ on $M$, we construct a Cartan system $(P, C(S))$ in such a way that $(P$, $C(S)$ ) is a defining Cartan system of $\mathbb{S}(S)$ (Theorem 2.3). Then, using $(P, C(S))$, we can study the structure of $(S)(S)$. Moreover, we prove the 
following theorem.

Theorem 2.2. Let $S_{1}$ and $S_{2}$ be Pfaffian systems on $M$. Then $S_{1}$ and $S_{2}$ are locallly equivalent under the action of \&s if and only if $\left(P, C\left(S_{1}\right)\right)$ and $\left(P, C\left(S_{2}\right)\right)$ are locally isomorphic.

This theorem together with the isomorphic reduction theorem of a Cartan system which is established in [7] gives us a principle to study the problem A). In particular, in Section 4.4, we carry out the classification of second order ordinary differential equations $y^{\prime \prime}=f\left(x, y, y^{\prime}\right)$ under the action of certain Lie pseudogroup.

In Section 3, we shall define a normal Cartan subsystem $\left(P, C^{\sharp}\right)$ of $(P, C)$ and its quotient Cartan system $(P, C) /\left(P, C^{\sharp}\right)$. Then we prove an existence theorem (Theorem 3.3).

In Section 4, we shall study the problem of integration of a Pfaffian system $S$ on $P$ in the case when $S$ is generated by invariant 1 -forms of a Cartan system $(P, C)$. Such a system will be denoted by $(S,(P, C))$. Then, by using $C$, we define a special subsystem of $S$, which is called a normal covariant system of $(S, C)$ (Definition 4.3). $S$ is said to be irreducible if there is no normal covariant system of $(S, C)$. If $S$ admits a normal covariant system, we can use the results in Section 3. After describing our method of reduction, we obtain the following theorems.

Theorem 4.4. The problem of integration of $(S,(P, C))$ is reduced to that of the following two types of systems:

1) $\left(S_{\sharp},\left(P_{\sharp}, C_{\sharp}\right)\right)$, where $S_{\sharp}$ is completely integrable;

2) $\left(S_{*},\left(P_{*}, C_{*}\right)\right)$, where $S_{*}$ is irreducible.

Theorem 4.5. Assume that $k+1=\operatorname{rank} S \geqq 2$ and that there are normal covariant systems $S_{i}$ of $(S, C), 1 \leqq i \leqq k$, satisfying the following conditions:

1) $\operatorname{rank} S_{i}=i, 1 \leqq i \leqq k$;

2) $S_{1} \subset S_{2} \subset \cdots \subset S_{k} \subset S$.

Then the integration of $S$ is generically reduced to that of some completely integrable Pfaffian systems.

For the integration of an irreducible system $\left(S_{*},\left(P_{*}, C_{*}\right)\right)$, we can also use the Cartan system $\left(P_{*}, C_{*}\right)$. In many cases, the structure equation of $\left(P_{*}, C_{*}\right)$ enables us to find another kind of useful subsystems of $S$. We shall deal with such subsystems in a forthcoming paper. 
Theorem 4.5 may be applicable to some special types of partial differential equations (see [4] $\S 34-37$ ).

Throughout this paper, all manifolds and mappings (functions) are assumed to be differentiable of class $C^{\infty}$ and the adjective "differentiable" is omitted unless otherwise stated. The theory is, however, also well applied to the real analytic case.

The author would like to express his sincere gratitude to Professor A. Morimoto for his kind advice and encouragement.

\section{$\S 1$. Cartan systems and Lie pseudogroups}

1.1. Let $M$ be a manifold. We denote by $\mathfrak{F}(M)$ the ring of all functions on $M$ and by $\wedge^{1}(M)$ the $\mathfrak{\mho}(M)$-module of all 1 -forms on $M$. An $\widetilde{F}(M)$-submodule $S$ of $\wedge^{1}(M)$ is called a Pfaffian system of rank $s$ on $M$ if there are $s$-forms $\theta^{1}, \cdots, \theta^{s}$ on $M$ such that

1) $S$ is generated by $\theta^{1}, \cdots, \theta^{s}$;

2) $\theta^{1}, \cdots, \theta^{s}$ are linearly independent at each point of $M$.

We denote it by $S=\left\langle\theta^{1}, \cdots, \theta^{s}\right\rangle . \quad\left(\theta^{1}, \cdots, \theta^{s}\right)$ is called a generator of $S$. A function $f$ on $M$ is called a first integral of $S$ if $d f \in S$. A submanifold $N$ of $M$ is called an integral manifold of $S$ if $\iota^{*} \theta=0$ for any $\theta \in S$, where $\iota ; N \rightarrow M$ is the inclusion. $S$ is said to be completely integrable if $d \theta \equiv 0(\bmod S)$ for any $\theta \in S$.

Let $\mathbb{S}(M)$ denote the set of all local transformations of $M$. For each $f \in \mathfrak{S}(M)$, we denote by $U(f)$ the domain of $f$ and by $V(f)$ the range of $f$. Let $\& 5$ be a pseudogroup on $M$ (for the precise definition, see [7] or [11]). For an open set $U$ of $M$, we denote by $\& \mid U$ the pseudogroup on $U$ consisting of all elements $f$ of $\&$ such that $U(f)$ and $V(f)$ are contained in $U$.

Let $P$ be a manifold and let $C=\left(u^{1}, \cdots, u^{n} ; \omega^{1}, \cdots, \omega^{r}\right)$ be a system of functions $u^{i}$ and 1 -forms $\omega^{j}$ on $P(n \geqq 0, r \geqq 1)$. For each $p \in P$, we denote by $D(C)_{p}$ the linear subspace of the tangent space $T_{p}(P)$ consisting of all vectors $X$ such that $\left.\omega^{j}(X)=0, X\right\rfloor d \omega^{j}=0,1 \leqq j \leqq r$, and $X u^{i}=0$, $1 \leqq i \leqq n$, where $X\rfloor d \omega^{j}$ is the inner derivative of $d \omega^{j}$ with respect to $X$. $C$ is called an invariant system on $P$ if it satisfies the following conditions:

1) $\omega^{1}, \cdots, \omega^{r}$ are linearly independent at each point of $P$;

2) the Pfaffian system $\left\langle\omega^{1}, \cdots, \omega^{r}\right\rangle$ is completely integrable;

3) $D(C)_{p}=0$ for any $p \in P$.

Then the pair $(P, C)$ is called a Cartan system. We denote by $\leftrightarrow S(P, C)$ 
the pseudogroup on $P$ consisting of all elements $f$ of $\varangle(P)$ such that $f^{*} \theta=\theta$ for every $\theta$ in $C$, where $f^{*} \theta$ is the pull-back of $\theta$ by $f$. A function $u$ (resp. a 1 -form $\omega$ ) on an open set $U$ of $P$ is called an invariant (resp. an invariant 1-form) of $(P, C)$ if it satisfies $f^{*} u=u$ (resp. $f^{*} \omega=\omega$ ) for any $f \in \mathbb{\leftrightarrow s}(P, C) \mid U$. A system of invariants of $(P, C), v^{1}, \cdots, v^{m}$, is called a complete system of invariants of $(P, C)$ if $v^{1}, \cdots, v^{m}$ are independent functions on $P$ and if, for any invariant $u$ of $(P, C)$, there is a function $F$ of $m$ variables such that $u=F\left(v^{1}, \cdots, v^{m}\right)$. Let $U$ be an open set of $P$. Restricting every member of $C$ to $U$, we get an invariant system $C \mid U$ on $U$.

Let $C_{i}=\left(u_{i}^{1}, \cdots, u_{i}^{n} ; \omega_{i}^{1}, \cdots, \omega_{i}^{r}\right)$ be an invariant system on a manifold $P_{i}(i=1,2)$. A diffeomorphism $F$ of $P_{1}$ onto $P_{2}$ is called an isomorphism of $\left(P_{1}, C_{1}\right)$ onto $\left(P_{2}, C_{2}\right)$ if it satisfies $F^{*} u_{1}^{j}=u_{2}^{j}, 1 \leqq j \leqq n$, and $F^{*} \omega_{2}^{k}=\omega_{1}^{k}$, $1 \leqq k \leqq r$. Let $p_{i} \in P_{i}(i=1,2)$. We say that $\left(P_{1}, C_{1}\right)$ and $\left(P_{2}, C_{2}\right)$ are locally isomorphic at $\left(p_{1}, p_{2}\right)$ if there are open neighborhoods $U_{i}$ of $p_{i}$, $i=1,2$, and an isomorphism of $\left(U_{1}, C_{1} \mid U_{1}\right)$ onto $\left(U_{2}, C_{2} \mid U_{2}\right)$ with $F\left(p_{1}\right)=$ $p_{2}$. For the equivalence problem of Cartan systems, we refer to [10].

Let $P$ and $M$ be manifolds and let $\pi: P \rightarrow M$ be a fibering (a surjective submersion). Let $E(\pi)$ denote the vertical distribution given by $E(\pi)_{p}=\operatorname{Ker}\left(\pi_{*}\right)_{p}, p \in P$, where $\left(\pi_{*}\right)_{p}$ is the differential of $\pi$ at $p$. An element $F$ of $\leftrightarrow(P)$ is called a prolongation (resp. a local prolongation) of an element $f$ of $₫(M)$ if it satisfies $(1) \pi(U(F))=U(f)$ (resp. $\pi(U(F)) \subset U(f)$ ) and (2) $\pi \circ F=f \circ \pi$ on $U(F)$.

Let $C$ be an invariant system on $P$ and let $\mathscr{S}_{0}(M, C)$ denote the set of all elements $f$ of $\mathbb{S S}(M)$ such that $f$ has a prolongation $F$ in $\mathbb{S}(P, C)$. We say that $(P, C)$ acts on $M$ with respect to $\pi$ if the following conditions are satisfied:

1) $E(\pi)$ is $\mathbb{S}(P, C)$-invariant, that is, $F_{*}\left(E(\pi)_{p}\right)=E(\pi)_{F(p)}$ for all $F \in$ $\circlearrowleft(P, C)$ and $p \in U(F)$;

2) for any $f \in \mathbb{S}_{0}(M, C)$ and any $p \in \pi^{-1}(U(f))$, there is an element $F$ of $\leftrightarrow(P, C)$ such that $p \in U(F)$ and $F$ is a local prolongation of $f$.

Moreover, we say that $(P, C)$ acts effectively on $M$ if $\pi$ satisfies the third condition:

3) if $\pi \circ F=\pi$ on $U(F), F \in \mathbb{S}(P, C)$, then $F=$ id. on $U(F)$.

We remark that if the system $(P, \pi, M)$ is a principal fibre bundle then, under a condition on $C$, we can verify the conditions 1 ), 2) and 3) without knowing $\&(P, C)$ explicitly. 
When $(P, C)$ acts on $M$, we denote by $₫ S(M, C)$ the pseudogroup on $M$ generated by $\mathbb{S}_{0}(M, C)$ (see [11] p. 8).

Proposition 1.1. Assume that $(P, C)$ acts on $M$ with respect to a fibering $\pi: P \rightarrow M$. Then, for any $f \in \mathbb{S}(M, C)$ and any $p \in \pi^{-1}(U(f))$, there is an element $F$ of $\leftrightarrow(P, C)$ such that $p \in U(F)$ and $F$ is a local prolongation of $f$.

For the proof, see [7] Proposition 1.1.

Let $C=\left(u^{1}, \cdots, u^{n} ; \omega^{1}, \cdots, \omega^{r}\right)$. Then the action of $(P, C)$ on $M$ is said to be normal if, for every open set $U$ of $M$ and every 1-form $\theta$ on $U$, there are functions $a_{j}$ on $\pi^{-1}(U), 1 \leqq j \leqq r$, such that $\pi^{*} \theta=\sum_{j=1}^{r} a_{j} \omega^{j}$ on $\pi^{-1}(U)$.

A pseudogroup $\mathbb{B S}$ on $M$ is called a Lie pseudogroup if there are a Cartan system $(P, C)$ and a fibering $\pi: P \rightarrow M$ such that $(P, C)$ acts effectively on $M$ with respect to $\pi$ and $\mathbb{S}=\mathbb{S}(M, C) .(P, C)$ is called a defining Cartan system of $\mathbb{S}$. We can construct a defining Cartan system of $\&$ in such a way that the action is normal. In many cases, the system $((P, C), \pi, M)$ can be considered as a geometric structure on $M$, such as a (higher order) $G$-structure or a Cartan connection.

1.2. Let $C=\left(u^{0}, \cdots, u^{n} ; \omega^{0}, \cdots, \omega^{r}\right)$ be an invariant system on a manifold $P$ and let $W$ be an open set of $P$. Suppose $\left(d u^{0}\right)_{p} \neq 0$ for any $p \epsilon$ $W$. For each $q \in W$, we define a submanifold of $W$ by

$$
Q(W, q)=\left\{p \in W ; u^{0}(p)=u^{0}(q)\right\} .
$$

Let $\iota: Q(W, q) \rightarrow P$ be the inclusion. Then we can define a system of functions and 1-forms on $Q(W, q)$ by

$$
C(W, q)=\left(\iota^{*} u^{1}, \cdots, \iota^{*} u^{n} ; \iota^{*} \omega^{1}, \cdots, \iota^{*} \omega^{r}\right) .
$$

It should be remarked that $C(W, q)$ is not necessarily an invariant system. In [7], we proved the following reduction theorems (for the terminologies "reducible" and "an $R$-system", see [7] § 2.1).

Theorem 1.2. Let $(P, C)$ and $W$ be as above. If $u^{0}$ is reducible on $W$, then, for any $q \in W,(Q(W, q), C(W, q))$ is a Cartan system.

THEOREM 1.3. Let $C_{i}=\left(u_{i}^{0}, \cdots, u_{i}^{n} ; \omega_{i}^{0}, \cdots, \omega_{i}^{r}\right)$ be an invariant system on a manifold $P_{i}(i=1,2)$. Assume that $\operatorname{dim} P_{1}=\operatorname{dim} P_{2}$ and that $u_{i}^{0}$ is reducible on an open set $W_{i}$ of $P_{i}(i=1,2)$. Let $q_{i} \in W_{i}(i=1,2)$. Then 
$\left(P_{1}, C_{1}\right)$ and $\left(P_{2} . C_{2}\right)$ are locally isomorphic at $\left(q_{1}, q_{2}\right)$ if and only if the following conditions are satisfied:

1) $u_{1}^{j}\left(q_{1}\right)=u_{2}^{j}\left(q_{2}\right)$ for all $i=0, \cdots, n$;

2) there are $R$-systems $\left(W_{i}^{\prime}, U, \Omega_{i}^{\alpha}, a_{j}, b^{k}, c_{m}^{l}\right)$ with $q_{i} \in W_{i}^{\prime}, i=1,2$;

$3)$ the Cartan systems $\left(Q\left(W_{1}, q_{1}\right), C\left(W_{1}, q_{1}\right)\right)$ and $\left(Q\left(W_{2}, q_{2}\right), C\left(W_{2}, q_{2}\right)\right)$ are locally isomorphic at $\left(q_{1}, q_{2}\right)$.

\section{$\S 2$. The equivalence problem of Pfaffian systems}

2.1. Let $M$ be a manifold and let $S$ be a Pfaffian system on an open set $U$ of $M$ (for the definition, see $\S 1.1$ ). Let $f$ be an element of $\&(M)$ satisfying $V(f)=U$. We denote by $f^{*} S$ the Pfaffian system on $U(f)$ consisting of all 1-forms $f^{*} \theta, \theta \in S$. Let $V$ be an open subset of $U$. We denote by $S \mid V$ the Pfaffian system on $V$ generated by all 1-forms $\theta \mid V, \theta \in S$, where $\theta \mid V$ is the restriction of $\theta$ to $V$. Let \&5 be a pseudogroup on $M$. We denote by $\leftrightarrow(S)$ the subset of $(\mathfrak{S} \mid U$ consisting of all elements $f \in \mathbb{S} \mid U$ such that $f^{*}(S \mid V(f))=S \mid U(f)$. $\quad \mathbb{S}(S)$ is a pseudogroup on $U$. We say that (3) leaves $S$ invariant if $\mathbb{( S}(S)=\left(\mathbb{S} \mid U\right.$. Let $S_{i}$ be a Pfaffian system on an open set $U_{i}$ of $M$ and let $x_{i} \in U_{i}(i=1,2)$.

Definition 2.1. We say that $S_{1}$ and $S_{2}$ are locally đs-equivalent at $\left(x_{1}, x_{2}\right)$ if there is an element $f$ of (s) such that

1) $x_{1} \in U(f) \subset U_{1}, x_{2} \in V(f) \subset U_{2}$;

2) $f\left(x_{1}\right)=x_{2}$;

3) $f^{*}\left(S_{2} \mid V(f)\right)=S_{1} \mid U(f)$.

Assume now that \&s is a Lie pseudogroup on $M$ (cf. $\S 1.1$ ). Let $(P, C), C=\left(u^{1}, \cdots, u^{n} ; \omega^{1}, \cdots, \omega^{r}\right)$, be a defining Cartan system of (s) with respect to a fibering $\pi: P \rightarrow M$. Let $S=\left\langle\theta^{1}, \cdots, \theta^{s}\right\rangle$ be a Pfaffian system of rank $s$ on an open set $U$ of $M$. Then we can define a Pfaffian system $S^{*}$ on $\pi^{-1}(U)$ by $S^{*}=\left\langle\pi^{*} \theta^{1}, \cdots, \pi^{*} \theta^{s}\right\rangle$. From now on, we assume that $s<r$.

Definition 2.2. Let $W$ be an open set of $\pi^{-1}(U)$ and let $u_{k}^{j}, 1 \leqq j \leqq$ $s, s+1 \leqq k \leqq r$, be functions on $W$. Then $\left(W ; u_{k}^{j}\right)$ is called a system of invariants of $(S, C)$ with respect to the ordering $\omega^{1}, \cdots, \omega^{r}$ if, putting

$$
\Omega^{j}=\omega^{j}+\sum_{k=s+1}^{r} u_{k}^{j} \omega^{k}, \quad 1 \leqq j \leqq s,
$$

$\left(\Omega^{1}, \cdots, \Omega^{s}\right)$ forms a generator of $S^{*} \mid W$.

Adding the functions $u_{k}^{i}, 1 \leqq j \leqq s, s+1 \leqq k \leqq r$, to $C \mid W$, we get an 
invariant system on $W$, which we shall denote by $C\left(W ; u_{k}^{j}\right)$. It is immediate to prove the following lemma.

LEMMA 2.1. Let $\left(W_{i} ; u_{k ; i}^{j}\right)$ be a system of invariants of $(S, C)$ with respect to the ordering $\omega^{1}, \cdots, \omega^{r}(i=1,2)$. If $W_{1} \cap W_{2} \neq \phi$, then $u_{k ; 1}^{j}=u_{k ; 2}^{j}$ on $W_{1} \cap W_{2}, 1 \leqq j \leqq s, s+1 \leqq k \leqq r$.

Definition 2.1. A system of invariants $\left(P^{*} ; u_{k}^{j}\right)$ of $(S, C)$ with respect to the ordering $\omega^{1}, \cdots, \omega^{r}$ is said to be maximal if, for any system of invariants $\left(W ; v_{k}^{j}\right)$ of $(S, C)$ with respect to the same ordering $\omega^{1}, \cdots, \omega^{r}$, we have $W \subset P^{*}$ (and hence $v_{k}^{j}=u_{k}^{j}$ on $W$ by Lemma 2.1 ).

Proposition 2.1. Let $\mathbb{S},(P, C)$ and $S$ be as above. Assume that the action of $(P, C)$ on $M$ is normal. Then, for any $q \in \pi^{-1}(U)$, there is a maximal system of invariants $\left(P^{*} ; u_{k}^{j}\right)$ of $(S, C)$ with $q \in P^{*}$.

Proof. By assumption, there are functions $a_{k}^{j}$ on $\pi^{-1}(U), 1 \leqq j \leqq s$, $1 \leqq k \leqq r$, such that $\pi^{*} \theta^{j}=\sum_{k=1}^{r} a_{k}^{j} \omega^{k}, 1 \leqq j \leqq s$. Since $\operatorname{rank}\left(a_{k}^{j}\right)=s$ on $\pi^{-1}(U)$, we can assume that the matrix $\left(a_{k}^{j}(q)\right)_{1 \leqq j, k \leqq s}$ is non-singular. Let $P^{*}$ denote the open subset of $\pi^{-1}(U)$ consisting of all points $p \in \pi^{-1}(U)$ such that the matrix $\left(a_{k}^{j}(p)\right)_{1 \leqq j, k \leqq s}$ is non-singular. Then we have $q \in P^{*}$. Let $\left(b_{k}^{j}(p)\right)_{1 \leqq j, k \leqq s}$ denote the inverse matrix of the above matrix. Set $u_{k}^{j}=$ $\sum_{n=1}^{s} b_{n}^{j} a_{k}^{n}$ on $P^{*}, 1 \leqq j \leqq s, s+1 \leqq k \leqq r$. Then it is easy to verify that $\left(P^{*} ; u_{k}^{j}\right)$ is a maximal system of invariants of $(S, C)$ with respect to the ordering $\omega^{1}, \cdots, \omega^{r}$.

Let $\&$ be a Lie pseudogroup on a manifold $M$ and let $(P, C), C=$ $\left(u^{1}, \cdots, u^{n} ; \omega^{1}, \cdots, \omega^{r}\right)$, be a defining Cartan system of \&s with respect to a fibering $\pi: P \rightarrow M$.

Theorem 2.2. Let $S_{i}$ be a Pfaffian system of rank $s$ on an open set $U_{i}$ of $M(i=1,2)$. Let $\left(P_{i}^{*} ; u_{k ; i}^{j}, 1 \leqq j \leqq s, s+1 \leqq k \leqq r\right)$ be a maximal system of invariants of $\left(S_{i}, C\right)$ with respect to the ordering $\omega^{1}, \cdots, \omega^{r}$ and let $x_{i} \in \pi\left(P_{i}^{*}\right)(i=1,2)$. Set $C_{i}^{*}=C\left(P_{i}^{*} ; u_{k ; i}^{j}\right)(i=1,2)$. Then $S_{1}$ and $S_{2}$ are locally (S)-equivalent at $\left(x_{1}, x_{2}\right)$ if and only if there are points $q_{i}$ of $P_{i}^{*}$ with $x_{i}=\pi\left(q_{i}\right), i=1,2$, such that $\left(P_{1}^{*}, C_{1}^{*}\right)$ and $\left(P_{2}^{*}, C_{2}^{*}\right)$ are locally isomorphic at $\left(q_{1}, q_{2}\right)$.

Theorem 2.3. Let $S$ be a Pfaffian system of rank $s$ on an open set $U$ of $M$. Let $\left(P^{*} ; u_{k}^{j}, 1 \leqq j \leqq s, s+1 \leqq k \leqq r\right)$ be a maximal system of invariants of $(S, C)$ with respect to the ordering $\omega^{1}, \cdots, \omega^{r}$. Suppose $\pi\left(P^{*}\right)=$ 
U. Set $C^{*}=C\left(P^{*} ; u_{k}^{j}\right)$. Then:

1) $\left(S(S)\right.$ is a Lie pseudogroup on $U$ and $\left(P^{*}, C^{*}\right)$ is a defining Cartan system of $\mathbb{S}(S)$ with respect to the fibering $\pi \mid P^{*}: P^{*} \rightarrow U$.

2) The Pfaffian system $S^{*} \mid P^{*}$ is generated by $s$ invariant 1-forms of $\left(P^{*}, C^{*}\right), \omega^{j}+\sum_{k=s+1}^{r} u_{k}^{j} \omega^{k}, 1 \leqq j \leqq s$.

3) If \&5 leaves $S$ invariant, then the functions $u_{k}^{j}, 1 \leqq j \leqq s, s+1 \leqq$ $k \leqq r$, are invariants of $(P, C)$.

The converse of 3 ) of Theorem 2.3 holds if $P^{*}=\pi^{-1}(U)$.

2.2. We shall prove the theorems.

Lemma 2.2. Let the situation and the notations be as in Theorem 2.2. Let $f$ be an element of $\&$ satisfying $U(f) \subset U_{1}$ and $V(f) \subset U_{2}$. Assume that $f$ has a prolongation $F$ in $\mathbb{S}(P, C)$.

1) If $U(F) \subset P_{1}^{*}$ and $f^{*}\left(S_{2} \mid V(f)\right)=S_{1} \mid U(f)$, then $V(F) \subset P_{2}^{*}$ and $F^{*} u_{k ; 2}^{j}=u_{k ; 1}^{j}$ on $U(F), 1 \leqq j \leqq s, s+1 \leqq k \leqq r$.

2) If $U(F) \subset P_{1}^{*}, V(F) \subset P_{2}^{*}$ and $F^{*} u_{k ; 2}^{j}=u_{k ; 1}^{j}$ on $U(F), 1 \leqq j \leqq s$, $s+1 \leqq k \leqq r$, then, for any $x \in U(f)$, there is an open neighborhood $V$ of $x$ in $U(f)$ such that $(f \mid V) *\left(S_{2} \mid W\right)=S_{1} \mid V$, where we put $W=f(V)$.

Proof. Let us fix a generator $\left(\theta_{i}^{1}, \cdots, \theta_{i}^{s}\right)$ of $S_{i}(i=1,2)$. By definition, putting

$$
\Omega_{i}^{j}=\omega^{j}+\sum_{k=s+1}^{r} u_{k ; i}^{j} \omega^{k}, \quad 1 \leqq j \leqq s,
$$

$\left(\Omega_{i}^{1}, \cdots, \Omega_{i}^{s}\right)$ forms a generator of $S_{i}^{*} \mid P_{i}^{*}(i=1,2)$.

1) If we set $\Omega^{j}=\left(F^{-1}\right)^{*} \Omega_{1}^{j}$ on $V(F), 1 \leqq j \leqq s$, then

$$
\Omega^{j}=\omega^{j}+\sum_{k=s+1}^{r}\left(\left(F^{-1}\right)^{*} u_{k ; 1}^{j}\right), \quad 1 \leqq j \leqq s .
$$

Using the condition $\left.f^{*}\left(S_{2} \mid V(f)\right)=S_{1} \mid U(f)\right)$, we can prove easily that $\left(\Omega^{1}\right.$, $\left.\cdots, \Omega^{s}\right)$ forms a generator of $S_{2}^{*} \mid V(F)$. This means that $\left(V(F) ;\left(F^{-1}\right)^{*} u_{k ; 1}^{j}\right)$ is a system of invariants of $\left(S_{2}, C\right)$ with respect to the ordering $\omega^{1}, \cdots, \omega^{r}$. Since $\left(P_{2}^{*} ; u_{k ; 2}^{j}\right)$ is maximal, we have $V(F) \subset P_{2}^{*}$ and hence $\left(F^{-1}\right)^{*} u_{k ; 1}^{j}=u_{k ; 2}^{j}$ for all $j$ and $k$.

2) By assumption, we have $F^{*} \Omega_{2}^{j}=\Omega_{1}^{j}$ on $U(F), 1 \leqq j \leqq s$. Since $\left(\Omega_{\imath}^{1}, \cdots, \Omega_{i}^{s}\right)$ is a generator of $S_{i}^{*} \mid P_{i}^{*}(i=1,2)$, there are functions $A_{k}^{j}$ on $U(F), 1 \leqq j, k \leqq s$, such that the matrix $\left(A_{k}^{j}(p)\right)$ is non-singular for any $p \in U(F)$ and

$$
F^{*}\left(\pi^{*} \theta_{2}^{j}\right)=\sum_{k=1}^{s} A_{k}^{j}\left(\pi^{*} \theta_{1}^{k}\right), \quad 1 \leqq j \leqq s
$$


Hence,

$$
\pi^{*}\left(f^{*} \theta_{2}^{j}\right)=\sum_{k=1}^{s} A_{k}^{j}\left(\pi^{*} \theta_{1}^{k}\right), \quad 1 \leqq j \leqq s .
$$

Take an open neighborhood $V$ of $x$ in $U(f)$ and a cross section $\rho: V \rightarrow$ $U(F)$ of the fibering $\pi \mid U(F): U(F) \rightarrow U(f)$. Then we have

$$
f^{*} \theta_{2}^{j}=\sum_{k=1}^{s}\left(\rho^{*} A_{k}^{j}\right) \theta_{1}^{k}, \quad 1 \leqq j \leqq s,
$$

on $V$ and hence $(f \mid V)^{*}\left(S_{2} \mid W\right)=S_{1} \mid V(W=f(V))$.

Proof of Theorem 2.2. Assume first that $S_{1}$ and $S_{2}$ are locally (S)equivalent at $\left(x_{1}, x_{2}\right)$. Then there is an element $f$ of $\mathbb{S}$ with the properties listed in Definition 2.1. Take a point $q_{1}$ of $P_{1}^{*}$ with $x_{1}=\pi\left(q_{1}\right)$. By Proposition 1.1, there is an element $F$ of $\circlearrowleft(P, C)$ such that $q_{1} \in U(F)$ and $F$ is a local prolongation of $f$. Shrinking $U(f)$ and $U(F)$ if necessary, we can assume that $U(F) \subset P_{1}^{*}$ and that $F$ is a prolongation of $f$. If we set $q_{2}=F\left(q_{1}\right)$, then $x_{2}=\pi\left(q_{2}\right)$. By Lemma 2.2-1), we can conclude that $F$ is an isomorphism of $\left(U(F), C_{1}^{*} \mid U(F)\right)$ onto $\left(V(F), C_{2}^{*} \mid V(F)\right)$.

Conversely, assume that there are open neighborhoods $W_{i}$ of $q_{i}$ in $P_{i}^{*}, i=1,2$, and an isomorphism $F$ of $\left(W_{1}, C_{1}^{*} \mid W_{1}\right)$ onto $\left(W_{2}, C_{2}^{*} \mid W_{2}\right)$ with $F\left(q_{1}\right)=q_{2}$. Then $F$ belongs to $\mathbb{S}(P, C)$. We can assume that $F$ is a prolongation of an element $f$ of (S). Using $F, f$ and Lemma 2.2-2), we can verify that $S_{1}$ and $S_{2}$ are locally (s)-equivalent at $\left(x_{1}, x_{2}\right)$.

Proof of Theorem 2.3. Let $\pi_{0}$ denote the restriction of $\pi$ to $P^{*}$. Since $\circlearrowleft\left(P^{*}, C^{*}\right) \subset \circlearrowleft \Im(P, C) \mid P^{*}$, the vertical distribution $E\left(\pi_{0}\right)$ is $\circlearrowleft\left(P^{*}, C^{*}\right)$ invariant. Take any $f \in \mathbb{S}_{0}\left(U, C^{*}\right)$ and any $x \in U(f)$. Then $f$ has a prolongation $F$ in $\mathbb{S}\left(P^{*}, C^{*}\right)$. It follows from Lemma 2.2-2) that there is an open neighborhood $V$ of $x$ in $U(f)$ such that $f \mid V \in \mathbb{S}(S)$. This implies that $\mathbb{S}_{0}\left(U, C^{*}\right) \subset \mathbb{S}(S)$. Next take any $f \in \mathbb{S}(S)$ and any $p \in \pi_{0}^{-1}(U(f))$. Then there is an element $F$ of $\mathbb{S}(P, C)$ such that $p \in U(F)$ and $F$ is a local prolongation of $f$. As before, we can assume that $U(F) \subset \pi_{0}^{-1}(U(f))$. Then, by Lemma $2.2-1), F$ belongs to $\&\left(P^{*}, C^{*}\right)$. Since $\mathbb{S}_{0}\left(U, C^{*}\right) \subset \mathbb{S}(S)$, this implies that $\left(P^{*}, C^{*}\right)$ acts on $U$ with respect to $\pi_{0}$. The above argument also shows that $\leftrightarrow\left(U, C^{*}\right)=\mathscr{\leftrightarrow}(S)$. The effectivity of this action is obvious. This proves 1). 2) may be obvious.

To show 3), it will be sufficient to prove that $₫(P, C) \mid P^{*}$ is contained in $\mathbb{S}\left(P^{*}, C^{*}\right)$. Take any $F \in \mathbb{S}(P, C) \mid P^{*}$ and any $p \in U(F)$. Then there are an open neighborhood $W$ of $p$ in $U(F)$ and an element $f$ of $\mathbb{E} \mid U$ such 
that $F \mid W$ is a prolongation of $f$. Since $\mathbb{S S}(S)=\mathbb{S} \mid U$, it follows from Lemma 2.2-1) that $F \mid W$ belongs to $\mathbb{S}\left(P^{*}, C^{*}\right)$. Since $\mathbb{S}\left(P^{*}, C^{*}\right)$ is a pseudogroup, we finally have $F \in \Theta\left(P^{*}, C^{*}\right)$. This completes the proof of Theorem 2.3.

\section{§3. Quotient Cartan systems}

3.1. Let $M$ be a manifold and let $E$ be a subset of the tangent bundle $T M$ of $M$. We say that $E$ is a $v$-family on $M$ if, for any $x \in M$, $E_{x}=E \cap T_{x}(M)$ is a linear subspace of $T_{x}(M)$. Therefore, a distribution on $M$ is a $v$-family $E$ with the following properties: (1) $\operatorname{dim} E_{x}=$ constant on $M$ and (2) $E$ is a differentiable subbundle of $T M$.

Let $E$ be a $v$-family on $M$ and let $Q$ be a set of 1 -forms on $M$. We define $v$-families on $M, A(Q), D(Q), H(Q)$, and a set of 1 -forms on $M$, $P(E)$, as follows.

$$
\begin{aligned}
& A(Q)_{x}=\left\{X \in T_{x}(M) ; \theta(X)=0, \theta \in Q\right\}, \\
& \left.D(Q)_{x}=\left\{X \in A(Q)_{x} ; X\right\rfloor d \theta=0, \theta \in Q\right\}, \\
& H(Q)_{x}=\left\{X \in A(Q)_{x} ; d \theta(X, Y)=0, \theta \in Q, Y \in A(Q)_{x}\right\}, \\
& P(E)=\left\{\theta \in \wedge^{1}(M) ; \theta(X)=0, X \in E\right\},
\end{aligned}
$$

$x \in M$. We have $D(Q)_{x} \subset H(Q)_{x} \subset A(Q)_{x}, x \in M$. Set $\operatorname{Ch}(Q)=P(H(Q))$. Then $\mathrm{Ch}(Q)$ is called the characteristic system of $Q$. It is easy to prove the following lemmas.

Lemma 3.1. Assume that $D(Q)$ (resp. $H(Q)$ ) is a distribution on $M$. Then $D(Q)$ (resp. $H(Q)$ ) is completely integrable. Moreover, let $x \in M$ and let $x^{1}, \cdots, x^{m}$ be independent first integrals of $D(Q)$ defined on a small open neighborhood $V$ of $x\left(m=\operatorname{dim} M-\operatorname{dim} D(Q)_{x}\right)$. Then, for every $\theta \in Q$, there are functions of $m$ variables $a_{\jmath}, 1 \leqq j \leqq m$, such that

$$
\theta=\sum_{j=1}^{m} a_{j}\left(x^{1}, \cdots, x^{m}\right) d x^{j} \quad \text { on } V \text {. }
$$

Lemma 3.2. Let $P$ be a manifold and let $\pi: P \rightarrow M$ be a fibering. Let $\pi^{*} Q$ denote the set of all 1-forms of the form $\pi^{*} \theta, \theta \in Q$. Then:

1) $E(\pi)_{p} \subset D\left(\pi^{*} Q\right)_{p}\left(E(\pi)_{p}=\operatorname{Ker}\left(\pi_{*}\right)_{p}, p \in P\right)$.

2) $\pi_{*}\left(H\left(\pi^{*} Q\right)_{p}\right)=H(Q)_{\pi(p)}(p \in P)$.

3) $\pi_{*}\left(D\left(\pi^{*} Q\right)_{p}\right)=D(Q)_{\pi(p)}(p \in P)$.

Let $C=\left(u^{1}, \cdots, u^{n} ; \omega^{1}, \cdots, \omega^{r}\right)$ be an invariant system on a manifold $P$ and let $S=\left\langle\omega^{1}, \cdots, \omega^{s_{0}}\right\rangle\left(s_{0} \leqq r\right)$ be a Pfaffian system on $P$. Set $H=$ 
$\left\{u^{1}, \cdots, u^{n}\right\}, Q=\left\{\omega^{1}, \cdots, \omega^{r}\right\}, Q_{0}=\left\{\omega^{1}, \cdots, \omega^{s 0}\right\}$ and $E^{0}=A\left(Q_{0}\right)$. We define $v$-families on $P, E^{i}, i \geqq 0$, and subsets of $Q, Q_{i}, i \geqq 0$, by induction. Suppose that $E^{i-1}$ and $Q_{i-1}$ are defined for an integer $i \geqq 1$. Then we set $E^{i}=D\left(Q_{i-1}\right)$ and $Q_{i}=Q \cap P\left(E^{i}\right)$. We have $E^{i} \supset E^{i+1}$ and $Q_{i} \subset Q_{i+1}(i \geqq$ $0)$. Set $E_{p}^{\infty}=\bigcap_{i \geqq 0} E_{p}^{i}(p \in P), Q_{\infty}=\bigcup_{i \geqq 0} Q_{i}$ and

$$
H_{\infty}=\left\{u \in H ; X u=0, X \in E^{\infty}\right\} .
$$

Suppose $H_{\infty}=\left\{u^{1}, \cdots, u^{m}\right\}$ and $Q_{\infty}=\left\{\omega^{1}, \cdots, \omega^{s}\right\}\left(m \leqq n, s_{0} \leqq s \leqq r\right)$. Finally, we set $S_{\infty}=\left\langle\omega^{1}, \cdots, \omega^{s}\right\rangle$.

Definition 3.1. $S$ is said to be closed in $C$ if, for each $i \geqq 0, E^{i}$ is a distribution on $P$ and if $S_{\infty}$ is completely integrable.

Definition 3.2. Let $C_{0}=\left(\bar{u}^{1}, \cdots, \bar{u}^{m} ; \bar{\omega}^{1}, \cdots, \bar{\omega}^{s}\right)$ be an invariant system on a manifold $P_{0}$. Then $\left(P_{0}, C_{0}\right)$ is called a quotient Cartan system of $(P, C)$ by $S$ if there is a fibering $\pi: P \rightarrow P_{0}$ such that

1) $E_{p}^{\infty}=E(\pi)_{p}$ for any $p \in P$;

2) $\pi^{*} \bar{u}^{i}=u^{i}, 1 \leqq i \leqq m$, and $\pi^{*} \bar{\omega}^{j}=\omega^{j}, 1 \leqq j \leqq s$.

In this case, we say that $S$ admits a quotient Cartan system $\left(P_{0}, C_{0}\right)=$ $(P, C) / S$.

As for the uniqueness, we can prove easily the following.

Propositron 3.1. Let $\left(P_{i}, C_{i}\right), C_{i}=\left(\bar{u}_{i}^{1}, \cdots, \bar{u}_{i}^{m} ; \bar{\omega}_{i}^{1}, \cdots, \bar{\omega}_{i}^{s}\right)$, be a quotient Cartan system of $(P, C)$ by $S$ with fibering $\pi_{i}(i=1,2)$. Then, for any $p \in P,\left(P_{1}, C_{1}\right)$ and $\left(P_{2}, C_{2}\right)$ are locally isomorphic at $\left(\pi_{1}(p), \pi_{2}(p)\right)$.

Let $v^{1}, \cdots, v^{s_{0}}$ be functions on $P$. Set

$$
C^{\sharp}=\left(u^{1}, \cdots, u^{n}, v^{1}, \cdots, v^{s_{0}} ; \omega^{1}, \cdots, \omega^{r}\right) .
$$

Definition 3.3. $\left(P, C^{\#}\right)$ is called a normal Cartan subsystem of $(P, C)$ if there are $s_{0} 1$-forms in $C$, say $\omega^{1}, \cdots, \omega^{s_{0}}$, such that

1) $S=\left\langle\omega^{1}, \cdots, \omega^{s 0}\right\rangle$ is completely integrable;

2) $v^{1}, \cdots, v^{s_{0}}$ are independent first integrals of $S$. $S$ is called the defining Pfaffian system of $\left(P, C^{\sharp}\right)$.

Assume that there exists a quotient Cartan system $\left(P_{0}, C_{0}\right)$ of $(P, C)$ by $S$ with fibering $\pi: P \rightarrow P_{0}$.

Definition 3.4. $\left(P_{0}, C_{0}\right)$ is called a quotient Cartan system of $(P, C)$ by $\left(P, C^{\sharp}\right)$ if the following conditions are satisfied: 
1) $G\left(P, C^{\sharp}\right)=\{F \in G(P, C) ; \pi \circ F=\pi$ on $U(F)\}$;

2) $(P, C)$ acts on $P_{0}$ with respect to $\pi$ and $\&\left(P_{0}, C_{0}\right)=\circlearrowleft\left(P_{0}, C\right)$.

In this case, we say that $\left(P, C^{\sharp}\right)$ admits a quotient Cartan system $\left(P_{0}, C_{0}\right)=$ $(P, C) /\left(P, C^{\sharp}\right)$.

3.2. We shall prove the following existence theorems. Let $(P, C)$, $C=\left(u^{1}, \cdots, u^{n} ; \omega^{1}, \cdots, \omega^{r}\right)$, be a Cartan system and let $S=\left\langle\omega^{1}, \cdots, \omega^{s_{0}}\right\rangle$. We use the notations in Section 3.1.

Theorem 3.2. Assume that $S$ is closed in $C$. Then, for any $p \in P$, there is an open neighborhood $P^{\prime}$ of $p$ such that $S \mid P^{\prime}$ admits a quotient Cartan system $\left(P^{\prime}, C \mid P^{\prime}\right) /\left(S \mid P^{\prime}\right)$.

Theorem 3.3. Let $\left(P, C^{\sharp}\right), C^{\#}=\left(u^{1}, \cdots, u^{n}, v^{1}, \cdots, v^{s_{0}} ; \omega^{1}, \cdots, \omega^{r}\right)$, be $a$ normal Cartan subsystem of $(P, C)$ with defining Pfaffian system $S$. Assume that the following conditions are satisfied:

1) $S$ is closed in $C$;

2) $d u^{i} \equiv 0\left(\bmod . \omega^{1}, \cdots, \omega^{s}\right), 1 \leqq i \leqq n$;

3) Let $t=\operatorname{dim} E_{p}^{\infty}-(r-s)(p \in P)$. Then $t \geqq 0$ and there are $t 1$ forms $\omega^{r+1}, \cdots, \omega^{r+t}$ on $P$ and functions $C_{j k}^{i}$ of $n$ variables, $s+1 \leqq i \leqq$ $r+t, 1 \leqq j, k \leqq r+t$, such that

a) If $X \in E^{\infty}$ satisfies $\omega^{i}(X)=0, s+1 \leqq i \leqq r+t$, then $X=0$;

b) $C_{j k}^{i}+C_{k j}^{i}=0$ for all $i, j, k$ and

$$
d \omega^{i}=\frac{1}{2} \sum_{j, k=1}^{r+t} C_{j k}^{i}\left(u^{1}, \cdots, u^{n}\right) \omega^{j} \wedge \omega^{k}, \quad s+1 \leqq i \leqq r+t .
$$

Then, for any $p \in P$, there is an open neighborhood $P^{\prime}$ of $p$ such that $\left(P^{\prime}\right.$, $\left.C^{\sharp} \mid P^{\prime}\right)$ admits a quotient Cartan system $\left(P^{\prime}, C \mid P^{\prime}\right) /\left(P^{\prime}, C^{\sharp} \mid P^{\prime}\right)$.

Proof of the theorems. Suppose first that $S$ is closed in $C$. Let $d \geqq 0$ be the smallest integer satisfying $Q_{\infty}=Q_{d}$. Then we have $E^{i}=E^{d+1}$ for all $i \geqq d+1$ and hence $D\left(Q_{\infty}\right)=E^{\infty}$. Let us set $h=\operatorname{dim} P-\operatorname{dim} E_{p}^{\infty}$. Since $E^{\infty}$ is completely integrable, we can take independent first integrals $x^{1}, \cdots, x^{h}$ of $E^{\infty}$ defined on a small open neighborhood $P^{\prime}$ of $p$. Let $R^{h}$ be the standard euclidean space of dimension $h$. Let $\pi: P^{\prime} \rightarrow R^{h}$ denote the mapping given by $\pi(q)=\left(x^{1}(q), \cdots, x^{h}(q)\right) \quad\left(q \in P^{\prime}\right)$. We can assume that $\pi$ is a fibering of $P^{\prime}$ onto an open set $P_{0}$ of $R^{h}$. Then, $E^{\infty}=E(\pi)$ on $P^{\prime}$. By Lemma 3.1, there are functions $\bar{u}^{i}, 1 \leqq i \leqq m$, and 1 -forms $\bar{\omega}^{j}$, $1 \leqq j \leqq s$, on $P_{0}$ such that $\pi^{*} \bar{u}^{i}=u^{i}, 1 \leqq i \leqq m$, and $\pi^{*} \bar{\omega}^{j}=\omega^{j}, 1 \leqq j \leqq s$. 
By assumption, $\left\langle\bar{\omega}^{1}, \cdots, \bar{\omega}^{s}\right\rangle$ is completely integrable. Set $C^{\prime}=\left(u^{1}, \cdots, u^{m}\right.$; $\left.\omega^{1}, \cdots, \omega^{s}\right)$ and $C_{0}=\left(\bar{u}^{1}, \cdots, \bar{u}^{m} ; \bar{\omega}^{1}, \cdots, \bar{\omega}^{s}\right)$. Then, from Lemma 3.2, we have $\pi_{*}\left(D\left(C^{\prime}\right)_{q}\right)=D\left(C_{0}\right)_{\pi(q)}$ for any $q \in P^{\prime}$ (see $\S 1.1$ ). Since $D\left(C^{\prime}\right)=D\left(Q_{\infty}\right)$, we finally have $D\left(C_{0}\right)_{x}=0$ for any $x \in P_{0}$. Therefore $\left(P_{0}, C_{0}\right)$ is a Cartan system. This completes the proof of Theorem 3.2.

Next suppose that the conditions of Theorem 3.3 are satisfied. We shall prove 1) of Definition 3.4. For this purpose, we first show how to construct $x^{1}, \cdots, x^{h}$. Notice that $v^{1}, \cdots, v^{s_{0}}$ are first integrals of $E^{\infty}$. Set $v_{0}^{i}=v^{i}, 1 \leqq i \leqq s_{0}$, and $s_{j}=\operatorname{dim}\left(E_{p}^{j-1} / E_{p}^{j}\right), j \geqq 1$. Let $k$ be an integer with $0 \leqq k \leqq d$. Assume that we obtain independent first integrals of $E^{k}$ on $P^{\prime}, v_{0}^{1}, \cdots, v_{0}^{s_{0}}, \cdots, v_{k}^{1}, \cdots, v_{k}^{s_{k}}$. Set $Q_{k}=\left\{\omega^{1}, \cdots, \omega^{t_{k}}\right\}\left(t_{k} \leqq s\right)$. Since $Q_{k} \subset$ $P\left(E^{k}\right)$, there are functions $A_{l}^{i j}$ on $P^{\prime}, 0 \leqq i \leqq k, 1 \leqq j \leqq t_{k}, 1 \leqq l \leqq s_{i}$, such that

$$
\omega^{j}=\sum_{l=0}^{k} \sum_{l=1}^{s_{2}} A_{l}^{i \jmath} d v_{\imath}^{l}, \quad 1 \leqq j \leqq t_{k} .
$$

Then it is not hard to see that $A_{l}^{i j}$ 's are first integrals of $E^{k+1}$. If $k<d$ or $E^{\infty} \subseteq E^{d}$, we can choose independent functions $v_{k+1}^{j}$ on $P^{\prime}, 1 \leqq j \leqq s_{k+1}$, in such a way that

a) $v_{i}^{j}, 0 \leqq i \leqq k+1,1 \leqq j \leqq s_{i}$, are independent on $P^{\prime}$;

b) for each $j, 1 \leqq j \leqq s_{k+1}, v_{k+1}^{j}$ coincides with some $A_{l}^{i j}$.

Then $v_{i}^{j}, 0 \leqq i \leqq k+1,1 \leqq j \leqq s_{i}$, are first integrals of $E^{k+1}$. We can repeat this procedure. In this way, renumbering $v_{i}^{j}$, we obtain $x^{1}, \cdots, x^{h}$.

Now take any $F \in \mathbb{S}\left(P^{\prime}, C^{\sharp} \mid P^{\prime}\right)$. Then, $F^{*} v_{0}^{j}=v_{0}^{j}, 1 \leqq j \leqq s_{0}$. Assume that $F$ satisfies $F^{*} v_{i}^{j}=v_{i}^{j}, 0 \leqq i \leqq k, 1 \leqq j \leqq s_{i}$. Then we have $F^{*} A_{l}^{i \jmath}=$ $A_{l}^{i j}$ for all $i, j$ and $l$ and hence $F^{*} v_{k+1}^{j}=v_{k+1}^{j}, 1 \leqq j \leqq s_{k+1}$. Thus, by induction, we can prove $F^{*} x^{j}=x^{j}, 1 \leqq j \leqq h$. This implies that $\pi \circ F=\pi$ on $U(F)$. The converse relation may be obvious.

Finally, we prove 2) of Definition 3.4. Since $E(\pi)=E^{\infty}$, it follows from Lemma 3.2 that $E(\pi)$ is $\left(S\left(P^{\prime}, C \mid P^{\prime}\right)\right.$-invariant. It is easy to verify that $\mathbb{S}_{0}\left(P_{0}, C \mid P^{\prime}\right) \subset \mathbb{S}\left(P_{0}, C_{0}\right)$ (see $\S 1.1$ ). In view of the proof of Theorem 2.3-1), it suffices to prove that, for any $f \in \mathbb{S}\left(P_{0}, C_{0}\right)$ and any $q_{1} \in \pi^{-1}(U(f))$, there is a local prolongation $F \in \mathbb{S}\left(P^{\prime}, C \mid P^{\prime}\right)$ of $f$ with $q_{1} \in U(F)$. Set $W_{1}=\pi^{-1}(U(f))$ and $W_{2}=\pi^{-1}(V(f))$. Let $\pi_{i}: W_{1} \times W_{2} \rightarrow W_{i}$ be the natural projection $(i=1,2)$. Set $U^{i}=\pi_{1}^{*} u^{i}-\pi_{2}^{*} u^{i}, 1 \leqq i \leqq n$, and, $\theta^{j}=\pi_{1}^{*} \omega^{j}-$ $\pi_{2}^{*} \omega^{j}, 1 \leqq j \leqq r+t$. We should integrate the exterior differential system $\Sigma$ on $W_{1} \times W_{2}$ generated by $U^{1}, \cdots, U^{n}, \theta^{1}, \cdots, \theta^{r}$ (cf. [5]). Let us consider the submanifold $N$ of $U(f) \times V(f)$ given by 


$$
N_{0}=\{(x, f(x)) \in U(f) \times V(f) ; \quad x \in U(f)\}
$$

and the submanifold $N=(\pi \times \pi)^{-1}\left(N_{0}\right)$ of $W_{1} \times W_{2}$. Then we have $\operatorname{dim} N=$ $\operatorname{dim} P+(r-s+t)$. Let $S^{\prime}$ denote the Pfaffian system on $N$ given by $S^{\prime}=\left\langle\iota^{*} \theta^{s+1}, \cdots, \iota^{*} \theta^{r+t}\right\rangle$, where $\iota: N \rightarrow W_{1} \times W_{2}$ is the inclusion. Since $U^{i}=0,1 \leqq i \leqq n$, and $\theta^{j}=0,1 \leqq j \leqq s$, on $N$, the condition 3 )-b) of Theorem 3.3 implies that $S^{\prime}$ is completely integrable. For the given point $q_{1}$, there is a point $q_{2}$ of $W_{2}$ such that $\left(q_{1}, q_{2}\right) \in N$. Let $L$ be the leaf of $S^{\prime}$ with $\left(q_{1}, q_{2}\right) \in L . \quad L$ is an integral manifold of $\Sigma$ with $\operatorname{dim} L=\operatorname{dim} P$. Moreover, the condition 3)-a) implies that the differential $\left(\pi_{i} \mid L\right)_{*}: T_{z}(L) \rightarrow$ $T_{q_{i}}(P)\left(z=\left(q_{1}, q_{2}\right)\right)$ is non-singular $(i=1,2)$. So we can define a local transformation $F$ of $P^{\prime}$ by $F=\left(\pi_{2} \mid L\right) \circ\left(\pi_{1} \mid L\right)^{-1}$ on a small open neighborhood $V$ of $q_{1}$ in $W_{1}$. Clearly, we have $F^{*} u^{i}=u^{i}, 1 \leqq i \leqq n, F^{*} \omega^{j}=\omega^{j}$, $1 \leqq j \leqq r$, and $\pi \circ F=f \circ \pi$ on $V$. Thus $F$ is a desired element of $\mathbb{S}\left(P^{\prime}\right.$, $C \mid P^{\prime}$ ). This completes the proof of Theorem 3.3.

\section{$\S 4$. Integration of Pfaffian systems}

4.1. By the integration of a Pfaffian system $S$, we mean to find integral manifolds of $S$. Let $M$ be a manifold and let $\theta$ be a closed 1-form on $M$. A function $f$ on an open set $U$ of $M$ is called an integral of $\theta$ if $d f=\theta$ on $U$. By the language "quadrature", we mean to construct an integral of a closed 1-form. We first consider a completely integrable Pfaffian system $S$ of rank $s$ on $M$.

Definition 4.1. Let $\left(\theta^{1}, \cdots, \theta^{s}\right)$ be a generator of $S$ and let $\omega^{1}, \cdots, \omega^{s}$ be 1 -forms on $M$. We say that $\left(\theta^{1}, \cdots, \theta^{s}\right)$ is a solvable generator of $S$ with auxiliary 1-forms $\omega^{1}, \cdots, \omega^{s}$ if the following conditions are satisfied:

1) $d \theta^{1}=\omega^{1} \wedge \theta^{1}, d \omega^{1}=0$;

2) (if $s \geqq 2$ ) for all $k=2, \cdots, s$,

$$
d \theta^{k} \equiv \omega^{k} \wedge \theta^{k}, d \omega^{k} \equiv 0 \quad\left(\bmod \theta^{1}, \cdots, \theta^{k-1}\right) .
$$

Take an integral $g$ of $\omega^{1}$. Then we get a closed 1-form $\theta=e^{-g} \theta^{1}$. Take an integral of $\theta, f$, defined on an open set $U$ of $M$ and consider the submanifold $N$ of $U$ defined by $f=$ constant. If $s=1$, then $N$ is an integral manifold of $S$. If $s \geqq 2$, then $\theta^{1}=0, d \theta^{2}=\omega^{2} \wedge \theta^{2}$ and $d \omega^{2}=0$ on $N$. So we can repeat the above procedure. Thus we have the following proposition.

Proposition 4.1. Let $m=\operatorname{dim} M$. Assume that we find a solvable 
generator of $S$. Then every $(m-s)$-dimensional integral manifold of $S$ is locally constructed by quadratures.

This means that we can find $s$ independent first integrals of $S$ by quadratures. Let $\left(\theta^{1}, \cdots, \theta^{s}\right)$ be a generator of $S$. An element $\theta$ of $S$ is called an integral 1-form of $S$ if there are functions $A_{j k}$ on $M, 1 \leqq j<$ $k \leqq s$, such that $d \theta=\sum_{j<k} A_{\jmath k} \theta^{j} \wedge \theta^{k}$ (cf. [1]). Assume that the generator $\left(\theta^{1}, \cdots, \theta^{s}\right)$ consists of integral 1 -forms $\theta^{i}$ of $S, 1 \leqq i \leqq s$. Then we find some first integrals of $S$ by differentiations. After reducing all known first integrals to constants, we get a Pfaffian system $\tilde{S}=\left\langle\tilde{\theta}^{1}, \cdots, \tilde{\theta}^{t}\right\rangle$ satisfying

$$
d \tilde{\theta}^{i}=\frac{1}{2} \sum_{j, k=1}^{t} C_{j k}^{i} \tilde{\theta}^{j} \wedge \tilde{\theta}^{k}, \quad 1 \leqq i \leqq t,
$$

where $C_{j k}^{2}, 1 \leqq i, j, k \leqq t$, are constants with $C_{j k}^{i}+C_{k j}^{i}=0$ (see [1]). If these constants define a solvable Lie algebra, we can find a solvable generator of $\tilde{S}$ by algebraic operations.

4.2. Let $S$ be a Pfaffian system on a manifold $M$. $S$ is said to be regular if the characteristic system $\mathrm{Ch}(S)$ of $S$ is a Pfaffian system on $M$. In this case, $\mathrm{Ch}(S)$ is completely integrable (see $\S 3.1$ ). The following lemma is well-known (see [4] p. 1060 and [5] p. 52).

Lemma 4.1. Let $s=$ rank $S$. Assume that there exists an absolute parallelism $\theta^{1}, \cdots, \theta^{s}, \omega^{1}, \cdots, \omega^{t}$ on $M$ satisfying $S=\left\langle\theta^{1}, \cdots, \theta^{s}\right\rangle(s+t=$ $\operatorname{dim} M)$. Set

$$
d \theta^{i} \equiv \frac{1}{2} \sum_{j, k=1}^{t} F_{j k}^{i} \omega^{j} \wedge \omega^{k} \quad\left(\bmod \theta^{1}, \cdots, \theta^{s}\right), 1 \leqq i \leqq s,
$$

where $F_{j k}^{i}, 1 \leqq i \leqq s, 1 \leqq j, k \leqq t$, are functions on $M$ with $F_{j k}^{i}+F_{k j}^{i}=0$. Then:

1) As an $\mathfrak{F}(M)$-submodule of $\bigwedge^{1}(M), \mathrm{Ch}(S)$ is generated by $\theta^{1}, \cdots, \theta^{s}$ and $\sum_{k=1}^{t} F_{\jmath k}^{i} \omega^{k}, 1 \leqq i \leqq s, 1 \leqq j \leqq t$.

2) If $S$ is regular, then there are integral 1-forms $\bar{\theta}^{1}, \cdots, \bar{\theta}^{s}$ of $\mathrm{Ch}(S)$ such that $\left(\bar{\theta}^{1}, \cdots, \bar{\theta}^{s}\right)$ forms a generator of $S$.

Definition 4.2. Let (5) be a pseudogroup on $M$. A regular Pfaffian system $S^{\prime}$ on $M$ is called a covariant system of $S$ with respect to $\mathbb{S}$ if (s) $(S)$ leaves $S^{\prime}$ invariant.

In this case, $\mathrm{Ch}\left(S^{\prime}\right)$ is also a covariant system of $S$. In [3] and [4], 
E. Cartan used such covariant systems for the problem of integration of second order partial differential equations. We want to use them more effectively.

Assume that $(5)$ is a Lie pseudogroup. Consider a defining Cartan system $\left(P^{*}, C^{*}\right)$ of $\circlearrowleft(S)$ with respect to a fibering $\pi$ of $P^{*}$ onto $M$ (for example, we can take the Cartan system $\left(P^{*}, C^{*}\right)$ which appeared in Theorem 2.3). As before, for a Pfaffian system $\tilde{S}$ on $M$, we denote by $\tilde{S}^{*}$ the Pfaffian system on $P^{*}$ generated by all 1 -forms $\pi^{*} \theta, \theta \in \tilde{S}$. If $\tilde{S}$ is regular, then $\mathrm{Ch}(\tilde{S})^{*}=\mathrm{Ch}\left(\tilde{S}^{*}\right)$ by Lemma $\left.4.2-1\right)$. Now we can see that the Pfaffian system to be considered is given by $S^{*}$. Let $S^{\prime}$ be a covariant system of $S$ with respect to $\mathbb{S}^{\prime}$. Then $\left(S^{\prime}\right)^{*}$ is a covariant system of $S^{*}$ with respect to $\Theta^{*}\left(P^{*}, C^{*}\right)$. Assume that the action of $\left(P^{*}, C^{*}\right)$ on $M$ is normal. Then, by Proposition 2.1 and Theorem 2.3, the systems $S^{*}$, $\left(S^{\prime}\right)^{*}$ and $\mathrm{Ch}\left(S^{\prime}\right)^{*}$ are generated by invariant 1 -forms of $\left(P^{*}, C^{*}\right)$. Changing $C^{*}$ if necessary, we can assume that $\mathrm{Ch}\left(S^{\prime}\right)^{*}$ is generated by some 1 -forms in $C^{*}$.

By this consideration, we are led to the following problem. Being given a Cartan system $(P, C), C=\left(u^{1}, \cdots, u^{n} ; \omega^{1}, \cdots, \omega^{r}\right)$, study the problem of integration of a regular Pfaffian system $S$ on $P$ generated by invariant 1-forms of $(P, C)$. Such a system will be denoted by $(S,(P, C))$. As SS $(P, C)$ leaves $S$ invariant, we can consider covariant systems of $S$. However, we should restrict ourselves to the study of those covariant systems of $S$ which are constructed by the so-called rational operations.

Definition 4.3. A regular Pfaffian system $S^{\prime}$ of rank $t$ on $P$ is called a normal covariant system of $(S, C)$ if it satisfies the following conditions:

1) $S^{\prime} \subset S$ and $0<t<\operatorname{rank} S$;

2) $S^{\prime}$ is generated by $t$-forms $\sum_{j=1}^{r} a_{j}^{i} \omega^{j}, 1 \leqq i \leqq t$, where $a_{j}^{i}, 1 \leqq$ $i \leqq t, 1 \leqq j \leqq r$, are real constants;

3) $\mathrm{Ch}\left(S^{\prime}\right)$ is generated by some 1 -forms in $C$;

4) $\mathrm{Ch}\left(S^{\prime}\right)$ is closed in $C$ (see Definition 3.1);

5) $S^{\prime}=\mathrm{Ch}\left(S^{\prime}\right) \cap S$.

$S$ is said to be irreducible with respect to $(P, C)$ if there is no normal covariant system of $(S, C)$.

The structure equation of $(P, C)$ enables us to find normal covariant systems of $(S, C)$. Let $P^{\prime}$ be an open set of $P$. Then it is clear that $S^{\prime} \mid P^{\prime}$ is a normal covariant system of $\left(S\left|P^{\prime}, C\right| P^{\prime}\right)$. 
Theorem 4.2. Let $(P, C), S$ and $S^{\prime}$ be as above. Then, for any $p \in P$, there are Cartan systems $\left(P_{0}, C_{0}\right)$ and $\left(P_{1}, C_{1}\right)$ and a regular Pfaffian system $S_{0}^{\prime}$ on $P$ such that

1) $P_{1}$ is an open neighborhood of $p$ in $P$ and $C_{1}=C \mid P_{1}$;

2) $\left(P_{0}, C_{0}\right)$ is a quotient Cartan system of $\left(P_{1}, C_{1}\right)$ by $\mathrm{Ch}\left(S^{\prime}\right) \mid P_{1}$ with fibering $\pi: P_{1} \rightarrow P_{0}$;

3) $\left(S_{0}^{\prime}\right)^{*}=S^{\prime} \mid P_{1}$ with respect to the fibering $\pi$;

4) $S_{0}^{\prime}$ is generated by invariant 1-forms of $\left(P_{0}, C_{0}\right)$;

5) for a submanifold $N_{0}$ of $P_{0}, N=\pi^{-1}\left(N_{0}\right)$ is an integral manifold of $S^{\prime}$ if and only if $N_{0}$ is an integral manifold of $S_{0}^{\prime}$;

6) for a normal covariant system $S^{\prime \prime}$ of $\left(S \mid P_{1}, C_{1}\right)$ satisfying $S^{\prime \prime} \subset S^{\prime} \mid P_{1}$ and $0<\operatorname{rank} S^{\prime \prime}<\operatorname{rank} S^{\prime}$, there is a unique normal covariant system $S_{0}^{\prime \prime}$ of $\left(S_{0}^{\prime}, C_{0}\right)$ with $\left(S_{0}^{\prime \prime}\right)^{*}=S^{\prime \prime}$;

7) for a normal covariant system $S_{0}^{\prime \prime}$ of $\left(S_{0}^{\prime}, C_{0}\right), S^{\prime \prime}=\left(S_{0}^{\prime \prime}\right)^{*}$ is a normal covariant system of $\left(S \mid P_{1}, C_{1}\right)$ satisfying $S^{\prime \prime} \subset S^{\prime} \mid P_{1}$ and $0<\operatorname{rank} S^{\prime \prime}<$ $\operatorname{rank} S^{\prime}$.

It should be remarked that once $\mathrm{Ch}\left(S^{\prime}\right)$ is integrated we can construct the objects in Theorem 4.2 by the rational operations. However, we can determine $\operatorname{dim} P_{0}$ and the structure equation of $\left(P_{0}, C_{0}\right)$ without integration. From 6) of Theorem 4.2, we have the following corollary.

Corollary 4.3. Let $(P, C)$ and $S$ be as before. Assume that there are normal covariant systems $S_{i}$ of $(S, C), 1 \leqq i \leqq k$, satisfying the following conditions:

a) $0<\operatorname{rank} S_{i}<\operatorname{rank} S_{i+1}, 1 \leqq i \leqq k-1$ (if $k \geqq 2$ );

b) $S_{1} \subset S_{2} \subset \cdots \subset S_{k} \subset S$.

Then, for any $p \in P$, there are a sequence of Cartan systems

$$
\left(P_{k+1}, C_{k+1}\right) \stackrel{\pi_{k}}{\longrightarrow}\left(P_{k}, C_{k}\right) \longrightarrow \cdots \stackrel{\pi_{2}}{\longrightarrow}\left(P_{2}, C_{2}\right) \stackrel{\pi_{1}}{\longrightarrow}\left(P_{1}, C_{1}\right)
$$

and regular Pfaffian systems $\tilde{S}_{i}$ and $\tilde{S}_{i}^{\prime}$ on $P_{i}, 1 \leqq i \leqq k+1$, such that

1) $P_{k+1}$ is an open neighborhood of $p$ in $P$ and $C_{k+1}=C \mid P_{k+1}$;

2) $\tilde{S}_{k+1}=S\left|P_{k+1}, \tilde{S}_{k+1}^{\prime}=S_{k}\right| P_{k+1}$ and $\tilde{S}_{1}^{\prime}=\tilde{S}_{1}$;

3) $\operatorname{rank} \tilde{S}_{i}=\operatorname{rank} S_{i}, 1 \leqq i \leqq k$;

4) $\tilde{S}_{i}$ is generated by invariant 1 -forms of $\left(P_{i}, C_{i}\right), 1 \leqq i \leqq k+1$;

5) $\tilde{S}_{i}^{\prime}$ is a normal covariant system of $\left(\tilde{S}_{i}, C_{i}\right), 2 \leqq i \leqq k+1$;

6) $\left(P_{i}, C_{i}\right)$ is a quotient Cartan system of $\left(P_{i+1}, C_{i+1}\right)$ by $\operatorname{Ch}\left(\tilde{S}_{\imath+1}^{\prime}\right)$ with fibering $\pi_{i}: P_{i+1} \rightarrow P_{\imath}, 1 \leqq i \leqq k$; 
7) $\left(\tilde{S}_{i}\right)^{*}=\tilde{S}_{i+1}^{\prime}$ with respect to the fibering $\pi_{i}, 1 \leqq i \leqq k$.

Proof of Theorem 4.2. We use the notations in Section 3.1. We set $\operatorname{Ch}\left(S^{\prime}\right)=\left\langle\omega^{1}, \cdots, \omega^{s_{0}}\right\rangle$ and $\operatorname{Ch}\left(S^{\prime}\right)_{\infty}=\left\langle\omega^{1}, \cdots, \omega^{s}\right\rangle$. Moreover,

$$
Q=\left\{\omega^{1}, \cdots, \omega^{r}\right\}, Q_{\infty}=\left\{\omega^{1}, \cdots, \omega^{s}\right\} \quad \text { and } \quad E^{\infty}=D\left(Q_{\infty}\right) .
$$

By Theorem 3.2, there is an open neighborhood $P_{1}$ of $p$ in $P$ such that $\mathrm{Ch}\left(S^{\prime}\right) \mid P_{1}$ admits a quotient system $\left(P_{0}, C_{0}\right)$ with fibering $\pi: P_{1} \rightarrow P_{0}$. Let $C_{0}=\left(\bar{u}^{1}, \cdots, \bar{u}^{m} ; \bar{\omega}^{1}, \cdots, \bar{\omega}^{s}\right)(m \leqq n)$. Without loss of generality, we can assume that $P_{1}=P$. Before going further, we prove the following lemma.

LEMma 4.2. Let $\tilde{S}_{0}$ be a Pfaffian system on $P_{0}$ and let $\tilde{S}=\left(\tilde{S}_{0}\right) *$ Then $\mathrm{Ch}(\tilde{S})=\left\langle\omega^{1}, \cdots, \omega^{k}\right\rangle$ if and only if $k \leqq s$ and $\operatorname{Ch}\left(\tilde{S}_{0}\right)=\left\langle\bar{\omega}^{1}, \cdots, \bar{\omega}^{k}\right\rangle$.

Proof of Lemma 4.2. Suppose first Ch $(\tilde{S})=\left\langle\omega^{1}, \cdots, \omega^{k}\right\rangle$. From Lemma 3.2, we have $E^{\infty} \subset H(\tilde{S})$ and hence $Q \cap \operatorname{Ch}(\tilde{S}) \subset Q_{\infty}$. This implies that $\omega^{i}$ belongs to $Q_{\infty}, 1 \leqq i \leqq k$. Thus, $k \leqq s$ and $\pi^{*} \bar{\omega}^{i}=\omega^{i}, 1 \leqq i \leqq k$. Take any $X \in H(\tilde{S})$. Then, by Lemma 1.2 , we can write $X=\pi_{*} Y$ for some $Y \in H(\tilde{S})$. Then, $\bar{\omega}^{i}(X)=\omega^{i}(Y)=0,1 \leqq i \leqq k$. Thus, $\bar{\omega}^{i} \in \operatorname{Ch}\left(\tilde{S}_{0}\right), 1 \leqq i \leqq$ k. Next, for any $\bar{\omega} \in \operatorname{Ch}\left(\tilde{S_{0}}\right)$ and any $Y \in H(\tilde{S})$, we have $\left(\pi^{*} \bar{\omega}\right)(Y)=0$ and hence $\pi^{*} \bar{\omega} \in \mathrm{Ch}(\tilde{S})$. This means that $\bar{\omega}$ is an $\mathfrak{F}\left(P_{0}\right)$-linear combination of $\bar{\omega}^{1}, \cdots, \bar{\omega}^{k}$. This proves $\operatorname{Ch}\left(\tilde{S}_{0}\right)=\left\langle\bar{\omega}^{1}, \cdots, \bar{\omega}^{k}\right\rangle$.

The converse follows easily from the formula $\operatorname{Ch}(\tilde{S})=\operatorname{Ch}\left(\tilde{S}_{0}\right)^{*}$ (cf. Lemma 4.1-1)).

We return to the proof of Theorem 4.2. Since $S^{\prime} \subset \mathrm{Ch}\left(S^{\prime}\right)$, it follows from the condition 2) of Definition 4.3 that $S^{\prime}$ is generated by $t$-forms $\theta^{i}=\sum_{\jmath=1}^{s_{0}} b_{\jmath}^{i} \omega^{j}, 1 \leqq i \leqq t$, where $b_{j}^{i}$ 's are real constants. If we set

$$
\bar{\theta}^{i}=\sum_{\jmath=1}^{s_{0}} b_{j}^{i} \bar{\omega}^{j}, \quad 1 \leqq i \leqq t,
$$

then $\pi^{*} \bar{\theta}^{i}=\theta^{i}, 1 \leqq i \leqq t$. We can define a Pfaffian system on $P_{0}$ by $S_{0}^{\prime}=\left\langle\bar{\theta}^{1}, \cdots, \bar{\theta}^{t}\right\rangle$. Then we have $\left(S_{0}^{\prime}\right)^{*}=S^{\prime}$ and hence $\operatorname{Ch}\left(S_{0}^{\prime}\right)=\left\langle\bar{\omega}^{1}, \cdots\right.$, $\left.\bar{\omega}^{s 0}\right\rangle$ by Lemma 4.2. This proves 3) and 4) of Theorem 4.2. 5) may be obvious.

Let $S^{\prime \prime}$ be a normal covariant system of $(S, C)$ with $S^{\prime \prime} \subset S^{\prime}$. Then, by the same argument as above, there is a unique Pfaffian system $S_{0}^{\prime \prime}$ on $P_{0}$ such that $\left(S_{0}^{\prime \prime}\right)^{*}=S^{\prime \prime}$ and $S_{0}^{\prime \prime}$ is generated by 1 -forms $\sum_{j=1}^{s} c_{j}^{i} \bar{\omega}^{j}, 1 \leqq$ $i \leqq \operatorname{rank} S^{\prime \prime}$, where $c_{j}^{i}$ 's are real constants. Now we prove 6) and 7). In view of the above remark and Lemma 4.2 , it will be sufficient to show that $\mathrm{Ch}\left(S^{\prime \prime}\right)=\left\langle\omega^{1}, \cdots, \omega^{k}\right\rangle(k \leqq s)$ is closed in $C$ if and only if $\mathrm{Ch}\left(S_{0}^{\prime \prime}\right)=$ 
$\left\langle\bar{\omega}^{1}, \cdots, \bar{\omega}^{k}\right\rangle$ is closed in $C_{0}$. Set $\tilde{Q}_{0}=\left\{\omega^{1}, \cdots, \omega^{k}\right\}$ and $\bar{Q}_{0}=\left\{\bar{\omega}^{1}, \cdots, \bar{\omega}^{k}\right\}$. Let $\left(\tilde{E}^{i}, \tilde{Q}_{i}\right)$ (resp. $\left.\left(\bar{E}^{i}, \bar{Q}_{i}\right)\right)$ be the system defined by Ch $\left(S^{\prime \prime}\right)$ (resp. Ch $\left(S_{0}^{\prime \prime}\right)$ ). Assume that $\pi^{*} \bar{Q}_{i-1}=\tilde{Q}_{i-1}$ for an integer $i \geqq 1$. Then, from Lemma 3.2, we have

$$
E^{\infty} \subset D\left(\pi^{*} \bar{Q}_{i-1}\right)=D\left(\tilde{Q}_{i-1}\right)=\tilde{E}^{i}
$$

and

$$
\pi_{*} \tilde{E}^{i}=\pi_{*}\left(D\left(\pi^{*} \bar{Q}_{i-1}\right)\right)=D\left(\bar{Q}_{i-1}\right)=\bar{E}^{i} .
$$

Take any $\bar{\omega} \in \bar{Q}_{i} \quad$ For any $X \in \tilde{E}^{i}$, we have $\left(\pi^{*} \bar{\omega}\right)(X)=0$ and hence $\pi^{*} \bar{\omega} \in$ $\tilde{Q}_{i}$. Next, take any $\omega \in \tilde{Q}_{i}$. Since $\tilde{Q}_{i} \subset Q_{\infty}$, there is a 1 -form $\bar{\omega}$ in $C_{0}$ such that $\omega=\pi^{*} \bar{\omega}$. Take any $Y \in \bar{E}^{i}$. We can write $Y=\pi_{*} X$ for some $X \in \tilde{E}^{i}$. Then we have

$$
\bar{\omega}(Y)=\bar{\omega}\left(\pi_{*} X\right)=\omega(X)=0
$$

and hence $\bar{\omega} \in \bar{Q}_{i}$. We have thereby proved $\pi^{*} \bar{Q}_{i}=\tilde{Q}_{i}$. Since $\pi^{*} \bar{Q}_{0}=\tilde{Q}_{0}$, we can prove, by induction, the following relations:

$$
E^{\infty} \subset \tilde{E}^{i}, \pi_{*} \tilde{E}^{i}=\bar{E}^{i}, \pi^{*} \bar{Q}_{i}=\tilde{Q}_{i} \quad(i \geqq 0) .
$$

Hence,

$$
E^{\infty} \subset \tilde{E}^{\infty}, \pi_{*} \tilde{E}^{\infty}=\bar{E}^{\infty}, \pi^{*} \bar{Q}_{\infty}=\tilde{Q}_{\infty} .
$$

Using these relations, we can easily verify that $\mathrm{Ch}\left(S^{\prime \prime}\right)$ is closed if and only if so is $\mathrm{Ch}\left(S_{0}^{\prime \prime}\right)$. This completes the proof of Theorem 4.2.

4.3. We are now in a position to study the problem of integration of a system $(S,(P, C))$, where $C=\left(u^{1}, \cdots, u^{n} ; \omega^{1}, \cdots, \omega^{r}\right)$ is an invariant system on a manifold $P$ and $S$ is a regular Pfaffian system on $P$ generated by invariant 1-forms of $(P, C)$. A normal covariant system $S^{\prime}$ of $(S, C)$ is said to be minimal if there is no normal covariant system $S^{\prime \prime}$ of $(S, C)$ satisfying $S^{\prime \prime} \subset S^{\prime}$ and rank $S^{\prime \prime}<\operatorname{rank} S^{\prime}$. From now on, we suppose that $S$ is neither completely integrable nor irreducible with respect to $(P, C)$.

Lemma 4.3. For each $p \in P$, there are an open neighborhood $P^{\prime}$ of $p$ and a minimal normal covariant system $S^{\prime}$ of $\left(S\left|P^{\prime}, C\right| P^{\prime}\right)$ such that, for any open neighborhood $U$ of $p$ in $P^{\prime}, S^{\prime} \mid U$ is also a minimal normal covariant system of $(S|U, C| U)$.

Proof. Let $S_{1}$ be a minimal normal covariant system of $(S, C)$. Assume that $S_{1}$ does not satisfy the property in Lemma 4.3. Then we can find an 
open neighborhood $P_{1}$ of $p$ and a minimal normal covariant system $S_{2}$ of $\left(S\left|P_{1}, C\right| P_{1}\right)$ satisfying $S_{2} \subset\left(S_{1} \mid P_{1}\right)$ and rank $S_{2}<$ rank $S_{1}$. Assume further that $S_{2}$ does not satisfy the property. Then we can proceed as above, and so on. Since the rank of $S$ is finite, we can repeat the procedure only finite number of times. Now Lemma 4.3 follows from this fact.

Let us fix a point $p$ of $P$ and take an open neighborhood $P^{\prime}$ of $p$ and a minimal normal covariant system $S^{\prime}$ of $\left(S\left|P^{\prime}, C\right| P^{\prime}\right)$ with the property stated in Lemma 4.3. Let $E^{\infty}$ be the distribution defined by $\mathrm{Ch}\left(S^{\prime}\right)$ (cf. §3.1). Set $s_{0}=\operatorname{rank} \operatorname{Ch}\left(S^{\prime}\right)$ and $h=\operatorname{dim} P-\operatorname{dim} E_{p}^{\infty}$. Assume that we can integrate $\mathrm{Ch}\left(S^{\prime}\right)$. Then we can choose $h$ independent first integrals $v^{1}, \cdots, v^{h}$ of $E^{\infty}$ defined on a small open neighborhood $P_{1}$ of $p$ in $P^{\prime}$ in such a way that $v^{1}, \cdots, v^{s_{0}}$ are first integrals of $\mathrm{Ch}\left(S^{\prime}\right)$ (see the proof of Theorem 3.3). Let $\pi: P_{1} \rightarrow R^{h}$ denote the mapping given by

$$
\pi(q)=\left(v^{1}(q), \cdots, v^{h}(q)\right) \quad\left(q \in P_{1}\right) .
$$

Then we can assume that $\pi$ is a fibering of $P_{1}$ onto a small open set $P_{0}$ of $\boldsymbol{R}^{h}$. Moreover, we can construct Cartan systems $\left(P_{0}, C_{0}\right)$ and $\left(P_{1}, C_{1}\right)$ and a regular Pfaffian system $S_{0}^{\prime}$ satisfying the properties listed in Theorem 4.2. By Theorem 4.2-7) and Lemma 4.3, $S_{0}^{\prime}$ is irreducible with respect to $\left(P_{0}, C_{0}\right)$.

Let $\left(x^{1}, \cdots, x^{h}\right)$ be the canonical coordinate system on $\boldsymbol{R}^{h}$ (and hence on $P_{0}$ ). Assume now that we can find an integral manifold $N_{0}$ of $S_{0}^{\prime}$ with defining equations $f^{i}\left(x^{1}, \cdots, x^{h}\right)=0,1 \leqq i \leqq m$. Let $N=\pi^{-1}\left(N_{0}\right)$ and let $S \mid N$ denote the $\widetilde{\mho}(N)$-submodule of $\Lambda^{1}(N)$ generated by all 1 -forms $\iota^{*} \theta$, $\theta \in S \mid P_{1}$, where $\iota: N \rightarrow P_{1}$ is the inclusion. Then it is clear that $N$ is defined by the equations $f^{i}\left(v^{1}, \cdots, v^{h}\right)=0,1 \leqq i \leqq m$, and that $S \mid N$ is finitely generated. Let us set

$$
C_{1}^{\#}=\left(u^{1}, \cdots, u^{n}, v^{1}, \cdots, v^{s_{0}} ; \omega^{1}, \cdots, \omega^{r}\right) .
$$

Then $\left(P_{1}, C_{1}^{\sharp}\right)$ is a normal Cartan subsystem of $\left(P_{1}, C_{1}\right)$ with defining Pfaffian system $\mathrm{Ch}\left(S^{\prime}\right) \mid P_{1}$. From the first part of the proof of Theorem 3.3, it can be seen that $v^{1}, \cdots, v^{h}$ are invariants of $\left(P_{1}, C_{1}^{\#}\right)$. So each function $f^{i}=f^{i}\left(v^{1}, \cdots, v^{h}\right)$ is an invariant of $\left(P_{1}, C_{1}^{\ddagger}\right)$. We want to get a Cartan system $(N, \tilde{C})$ so that $S \mid N$ is generated by invariant 1-forms of $(N, \tilde{C})$. For this purpose, we use Theorem 1.2. Carrying out the isomorphic reduction of $\left(P_{1}, C_{1}^{\sharp}\right)$ by the invariants $f^{i}, 1 \leqq i \leqq m$, we may get a Cartan system $(N, \tilde{C})$ (we reduce each $f^{i}$ to zero). $N_{0}$ (or $N$ ) is said to be generic 
if the above procedure really yields a Cartan system $(N, \tilde{C})$ and if $S \mid N$ is a regular Pfaffian system on $N$. In this case, $S \mid N$ is generated by invariant 1-forms of $(N, \tilde{C})$ because, for every invariant 1-form $\omega$ of $\left(P_{1}, C_{1}^{\sharp}\right), \iota^{*} \omega$ is an invariant 1 -form of $(N, \tilde{C})$. Thus, for a generic integral manifold $N_{0}$ of $S_{0}^{\prime}$, we get a system $(S \mid N,(N \mid \tilde{C}))$, where we put $N=\pi^{-1}\left(N_{0}\right)$. Let us arrange the above procedures as follows.

Step 1) For a point $p$ of $P$, find an open neighborhood $P^{\prime}$ of $p$ and a minimal normal covariant system $S^{\prime}$ of $\left(S\left|P^{\prime}, C\right| P^{\prime}\right)$ with the property stated in Lemma 4.3 .

Step 2) Find $s_{0}$ independent first integrals of $\mathrm{Ch}\left(S^{\prime}\right)$.

Step 3) Construct Cartan systems $\left(P_{0}, C_{0}\right)$ and $\left(P_{1}, C_{1}\right)$, a fibering $\pi$ : $P_{1} \rightarrow P_{0}$ and a regular Pfaffian system $S_{0}^{\prime}$ on $P_{0}$ satisfying the properties listed in Theorem 4.2 .

Step 4) Find an integral manifold $N_{0}$ of $S_{0}^{\prime}$ with defining equations $f^{i}\left(x^{1}, \cdots, x^{h}\right)=0,1 \leqq i \leqq m$.

Step 5) Carry out the isomorphic reduction of $\left(P_{1}, C_{1}^{\#}\right)$ by the invariants $f^{i}\left(v^{1}, \cdots, v^{h}\right), 1 \leqq i \leqq m$ (we reduce $f^{i}$ to zero).

Step 6) For a generic integral manifold $N_{0}$ of $S_{0}^{\prime}$, describe the system $(S \mid N,(N, \tilde{C}))$, where $N=\pi^{-1}\left(N_{0}\right)$.

If $S \mid N$ is neither completely integrable nor irreducible with respect to $(N, \tilde{C})$, then we go back to Step 1$)$. Notice that we can achieve Steps $1), 3), 5)$ and 6 ) by rational operations. Moreover, $S_{0}^{\prime}$ is irreducible with respect to $\left(P_{0}, C_{0}\right)$. As a general result, we have the following theorem. By the word "generically", we mean to consider, in Step 6), only generic integral manifolds of $S_{0}^{\prime}$.

Theorem 4.4. Let $(P, C)$ and $S$ be as before. Then the integration of $(S,(P, C))$ is generically reduced to that of the following two types of systems:

1) $\left(S_{\sharp},\left(P_{\#}, C_{\sharp}\right)\right)$, where $S_{\sharp}$ is completely integrable:

2) $\left(S_{*},\left(P_{*}, C_{*}\right)\right)$, where $S_{*}$ is irreducible with respect to $\left(P_{*}, C_{*}\right)$.

If all systems $\left(S_{*},\left(P_{*}, C_{*}\right)\right)$ of the second type satisfy the condition rank $S^{*}=1$, then the integration of $S$ is reduced to that of completely integrable Pfaffian systems.

Theorem 4.5. Let $(P, C)$ and $S$ be as before. Assume that

$$
k+1=\operatorname{rank} S \geqq 2
$$

and that there are normal covariant systems $S_{i}$ of $(S, C), 1 \leqq i \leqq k$, satis- 
fying the following conditions:

1) $\operatorname{rank} S_{i}=i, 1 \leqq i \leqq k$;

2) $S_{1} \subset S_{2} \subset \cdots \subset S_{k} \subset S$.

Then the integration of $S$ is generically reduced to that of finite number of completely integrable Pfaffian systems.

The meaning of the word "generically" will be clarified in the following proof.

Proof of Theorem 4.5. Suppose that $S$ is not completely integrable. First of all, we integrate completely integrable Pfaffian systems $\mathrm{Ch}\left(S_{i}\right)$, $1 \leqq i \leqq k$. Then, for each point $p$ of $P$, we can construct a sequence of Cartan systems

$$
\left(P_{k+1}, C_{k+1}\right) \stackrel{\pi_{k}}{\longrightarrow}\left(P_{k}, C_{k}\right) \longrightarrow \cdots \stackrel{\pi_{2}}{\longrightarrow}\left(P_{2}, C_{2}\right) \stackrel{\pi_{1}}{\longrightarrow}\left(P_{1}, C_{1}\right)
$$

and regular Pfaffian systems $\tilde{S}_{i}$ and $\tilde{S}_{i}^{\prime}$ on $P_{i}, 1 \leqq i \leqq k+1$, satisfying the properties listed in Corollary 4.3. Then, $\operatorname{rank} \tilde{S}_{i}=i, 1 \leqq i \leqq k+1$. Assume that we can find a generic integral manifold $N_{i}$ of $\tilde{S}_{i}$ for an integer $i(1 \leqq i \leqq k)$. Set $N_{i+1}=\pi_{i}^{-1}\left(N_{i}\right)$ and consider the Pfaffian system $S_{i}=\tilde{S}_{i+1} \mid N_{i+1}$. By assumption, we have rank $S_{i} \leqq 1$. If rank $S_{i}=0$, then $N_{i+1}$ is an integral manifold of $\tilde{S}_{i+1}$. So we have only to consider the case rank $S_{i}=1$. Since rank $\tilde{S}_{1}=1$, we are led to the integration of a regular Pfaffian system of rank $1, S_{*}$, on a manifold $P_{*}$. In this case, we can use a theorem of Frobenius and Darboux (see [12] pp. 137-141).

Take a generator $\theta$ of $S_{*}$. A point $x$ of $P_{*}$ is said to be generic if there is an open neighborhood $V$ of $x$ such that $P(D(\theta \mid V))$ is a Pfaffian system on $V$, where $\theta \mid V$ is the resriction of $\theta$ to $V$ (for the notation $P(D(\theta \mid V))$, see $\S 3.1)$. Consider a generic point $x$ of $P_{*}$ and an open neighborhood $V$ of $x$ satisfying the above condition. Notice that $\mathrm{Ch}\left(S_{*}\right)$ and $P(D(\theta \mid V))$ are completely integrable. Let $2 k+1=\operatorname{rank} \mathrm{Ch}\left(S_{*}\right)$. Then, by the theorem of Frobenius and Darboux, we can find independent first integrals of $\operatorname{Ch}\left(S_{*}\right), x^{1}, \cdots, x^{k}, z, p_{1}, \cdots, p_{k}$, and a first integral $u$ of $P(D(\theta \mid V))$ such that

$$
\begin{aligned}
& \theta=d z-\sum_{j=1}^{k} p_{j} d x^{j} \quad \text { for } \operatorname{rank} P(D(\theta \mid V))=2 k+1, \\
& \theta=u\left(d z-\sum_{j=1}^{k} p_{j} d x^{j}\right) \quad \text { for } \operatorname{rank} P(D(\theta \mid V))=2 k+2 .
\end{aligned}
$$

Using $x^{1}, \cdots, x^{k}, z, p_{1}, \cdots, p_{k}$, we can construct integral manifolds of $S_{*}$. This completes the proof of Theorem 4.5. 
The above 1-form $d z-\sum_{j=1}^{k} p_{j} d x^{j}$ is a standard form of generators of $S_{*}$. This can be generalized as follows. Let $S$ be a regular Pfaffian system of rank $s$ on a manifold $P$. Then a generator $\left(\theta^{1}, \cdots, \theta^{s}\right)$ of $S$ is called an integral generator of $S$ if $\theta^{i}$ is an integral 1-form of $\mathrm{Ch}(S), 1 \leqq$ $i \leqq s$. By Lemma 4.1-2), such a generator exists at least locally. Let $t=\operatorname{rank} \operatorname{Ch}(S)$. Assume that $s<t$ and that we can find an integral generator $\left(\theta^{1}, \cdots, \theta^{s}\right)$ of $S$ and $t$ independent first integrals $y^{1}, \cdots, y^{t}$ of $\mathrm{Ch}(S)$. Then each $\theta^{i}$ must be of the form:

$$
\theta^{i}=\sum_{j=1}^{t} A_{j}^{i}\left(y^{1}, \cdots, y^{t}\right) d y^{j} \quad(1 \leqq i \leqq s) .
$$

From this expression, we can get another integral generator $\left(\tilde{\theta}^{1}, \cdots, \tilde{\theta}^{s}\right)$ of $S$, where $\tilde{\theta}^{i}$ is given by

$$
\tilde{\theta}^{i}=d y^{i}-\sum_{j=s+1}^{t} B_{\jmath}^{i}\left(y^{1}, \cdots, y^{t}\right) d y, \quad 1 \leqq i \leqq s .
$$

This can be considered as a standard form of generators of $S$. Using such a standard form, we can often construct integral manifolds of $S$ (see [3] p. 159 and pp. 169-171).

Consider a system $(S,(P, C)$ ), where $S$ is irreducible with yespect to $(P, C)$. Then we should first try to find an integral generator of $S$ by integrating only completely integrable Pfaffian systems. If it is impossible, then we try to find a Cartan system $\left(P, C^{\prime}\right)$ so that $\left(S, C^{\prime}\right)$ admits a normal covariant system. Of course, this is not an effective method of integration of $S$.

Remark. Let us consider a general system $(S,(P, C))$. When we find integral manifolds of $S$ on which some fixed linearly independent 1-forms on $P$ are still linearly independent, there may appear in Step 4) and Step 6) certain integrability conditions. These are main obstructions for solving partial differential equations.

Let us illustrate it by a simple example. Consider a Cartan system $(P, C)$, where $\operatorname{dim} P=7$ and $C=\left(\omega^{1}, \cdots, \omega^{7}\right)$ with

$$
\left\{\begin{array}{l}
d \omega^{3}=\omega^{1} \wedge \omega^{4}+\omega^{2} \wedge \omega^{5}, \\
d \omega^{4}=\omega^{1} \wedge \omega^{6}+\omega^{2} \wedge \omega^{7}, \\
d \omega^{5}=\omega^{1} \wedge \omega^{7}-\omega^{2} \wedge \omega^{6}, \\
d \omega^{i}=0, \quad i=1,2,6,7 .
\end{array}\right.
$$

Set $S=\left\langle\omega^{3}, \omega^{4}, \omega^{5}\right\rangle$. Consider two-dimensional integral manifolds of $S$ on which $\omega^{1}$ and $\omega^{2}$ are still linearly independent. Let $S^{\prime}=\left\langle\omega^{5}\right\rangle$. Then, 
$\operatorname{Ch}\left(S^{\prime}\right)=\left\langle\omega^{1}, \omega^{2}, \omega^{5}, \omega^{6}, \omega^{7}\right\rangle$ and $\left(\omega^{1}, \omega^{2}, \omega^{6}, \omega^{7}, \omega^{5}\right)$ is a solvable generator of $\mathrm{Ch}\left(S^{\prime}\right)$. We can see that $S^{\prime}$ is a normal covariant system of $(S, C)$. Let $\left(P_{0}, C_{0}\right)$ be a quotient Cartan system of $(P, C)$ by $\mathrm{Ch}\left(S^{\prime}\right)$ with fibering $\pi$ : $P \rightarrow P_{0}$. Then, $\operatorname{dim} P_{0}=5$ and $C_{0}=\left(\bar{\omega}^{1}, \bar{\omega}^{2}, \bar{\omega}^{5}, \bar{\omega}^{6}, \bar{\omega}^{7}\right)$ with

$$
\left\{\begin{array}{l}
d \bar{\omega}^{5}=\bar{\omega}^{1} \wedge \bar{\omega}^{7}-\bar{\omega}^{2} \wedge \bar{\omega}^{6} \\
d \bar{\omega}^{i}=0, \quad i=1,2,6,7
\end{array}\right.
$$

$S_{0}^{\prime}$ is given by $S_{0}^{\prime}=\left\langle\bar{\omega}^{5}\right\rangle$. Let $N_{0}$ be a two-dimensional integral manifold of $S_{0}^{\prime}$ on which $\bar{\omega}^{1}$ and $\bar{\omega}^{2}$ are still linearly independent and let $N=\pi^{-1}\left(N_{0}\right)$. Then $N$ is generic. We get a Cartan $\operatorname{system}(N, \tilde{C})$, where $\operatorname{dim} N=4$ and $\tilde{C}=\left(u^{1}, u^{2} ; \theta^{1}, \theta^{2}, \theta^{3}, \theta^{4}\right)$ with

$$
\left\{\begin{array}{l}
d \theta^{1}=0 \\
d \theta^{2}=0 \\
d \theta^{3}=\theta^{1} \wedge \theta^{4}, \\
d \theta^{4}=u \theta^{1} \wedge \theta^{2} .
\end{array}\right.
$$

The invariant $u$ is a function of $u^{1}$ and $u^{2}$. We have $S \mid N=\left\langle\theta^{3}, \theta^{4}\right\rangle$. In this case, the integrability condition is given by $u=0$. If $u=0$, then $S \mid N$ is completely integrable and $\left(\theta^{4}, \theta^{3}\right)$ forms a solvable generator of $S \mid N$.

4.4. Finally, we consider the problem of classification. Let \&s be a Lie pseudogroup on a manifold $M$ and let $(P, C)$ be a defining Cartan system of (5) with respect to a fibering $\pi$ of $P$ onto $M$. Assume that the action is normal (cf. § 1.1). Let $S_{i}$ be a regular Pfaffian system on an open set $U_{i}$ of $M(i=1,2)$. Then we can consider the Cartan system $\left(P_{i}^{*}, C_{i}^{*}\right)$ which appeared in Theorem 2.2. $S_{i}$ determines a regular Pfaffian system $S_{i}^{*}$ on $P_{i}^{*}$, which is generated by invariant 1-forms of $\left(P_{i}^{*}, C_{i}^{*}\right)$ (Theorem 2.3-2)). If $S_{1}$ and $S_{2}$ are locally (S)-equivalent, then $\left(P_{i}^{*}, C_{i}^{*}\right)$ and $\left(P_{2}^{*}, C_{2}^{*}\right)$ are locally isomorphic (Theorem 2.2). Shrinking $U_{i}$ and $P_{i}^{*}$ if necessary, we can assume that there is an isomorphism $F$ of $\left(P_{1}^{*}\right.$, $\left.C_{1}^{*}\right)$ onto $\left(P_{2}^{*}, C_{2}^{*}\right)$ with $F^{*} S_{2}^{*}=S_{1}^{*}$. Then there is a one-to-one correspondence between the set of all normal covariant systems of $\left(S_{1}^{*},\left(P_{1}^{*}, C_{1}^{*}\right)\right)$ and that of $\left(S_{2}^{*},\left(P_{2}^{*}, C_{2}^{*}\right)\right)$. Therfore we can conclude that the integration of $S_{1}^{*}$ is formally equivalent to that of $S_{2}^{*}$. Thus we get a class of Pfaffian systems to which the same method of integration can be well applied.

We are interested in the problem of classification of all Pfaffian 
systems $S$ such that $\mathscr{S}(S)$ is finite dimensional. In this case, using Theorem 1.3 finite number of times, we can reduce the problem to the following lemma.

LEMma 4.4. Let $\left(P_{i}, C_{i}\right), C_{i}=\left(\omega_{\imath}^{1}, \cdots, \omega_{i}^{r}\right)$, be a Cartan system with $r=\operatorname{dim} P_{i}(i=1,2) . \quad$ Assume that there are constants $C_{k l ; i}^{j}, 1 \leqq j, k, l \leqq r$. satisfying $C_{k l, i}^{j}+C_{l k ; i}^{j}=0$ for all $j, k, l$ and

$$
d \omega_{i}^{l}=\frac{1}{2} \sum_{k, l=1}^{r} C_{k l ; i}^{j} \omega_{\imath}^{k} \wedge \omega_{i}^{l}, \quad 1 \leqq j \leqq r(i=1,2) .
$$

Then $\left(P_{1}, C_{1}\right)$ and $\left(P_{2}, C_{2}\right)$ are locally isomorphic at every point of $P_{1} \times P_{2}$ if and only if $C_{k l ; 1}^{j}=C_{k l ; 2}^{i}, 1 \leqq j, k, l \leqq r$.

For the proof, see [12] p. 221.

Now we deal with the problem of classification of second order ordinary differential equations

$$
\frac{d^{2} y}{d x^{2}}=f\left(x, y, \frac{d y}{d x}\right)
$$

under the action of the following Lie pseudogroup on $\boldsymbol{R}^{2}$.

$$
\text { (3)': }\left\{\begin{array}{l}
X=x+a, \\
Y=Y(x, y),
\end{array} \quad(a \in R)\right. \text {. }
$$

Let $(P, C)$ denote the Cartan system givən by

$$
\begin{gathered}
P=\left\{(x, y, p, q, r, s, t) \in R^{7} ; \quad q \neq 0\right\}, \\
C:\left\{\begin{array}{l}
\omega_{1}=d x, \\
\omega^{2}=q d y-p q d x, \\
\omega^{3}=-q d p+r \omega^{1}+s \omega^{2}, \\
\omega^{4}=d q / q+s \omega^{1}+t \omega^{2} .
\end{array}\right.
\end{gathered}
$$

Let $\pi: P \rightarrow R^{3}$ and $\pi^{\prime}: P \rightarrow R^{2}$ denote the natural projections defined by $\pi(z)=(x, y, p)$ and $\pi^{\prime}(z)=(x, y)$, respectively $(z \in P)$. Then $(P, C)$ is a defining Cartan system of (S)'. Moreover, $(P, C)$ acts effectively on $\boldsymbol{R}^{3}$ with respect to $\pi$. So it defines a Lie pseudogroup (S) on $\boldsymbol{R}^{3}$. Let $f$ be a function on an open set $U(f)$ of $\boldsymbol{R}^{3}$. Set $\theta=d y-p d x, \theta_{f}=d p-f(x, y, p) d x$ and $S_{f}=\left\langle\theta, \theta_{f}\right\rangle$. Then we are led to the problem of classification of the Pfaffian systems $S_{f}$ under the action of ㅇs. 
We have $q \pi^{*} \theta=\omega^{2}$ and $s q \pi^{*} \theta-q \pi^{*} \theta_{f}=\omega^{3}-u^{0} \omega^{1}$ on $P_{f}^{*}=\pi^{-1}(U(f))$, where we put $u^{0}=r-q f . \quad\left(P_{f}^{*} ; u^{0}\right)$ is a maximal system of invariants of $\left(S_{f}, C\right)$. Set $C_{f}^{*}=\left(u^{0} ; \omega^{1}, \cdots, \omega^{4}\right)$. Then we get a Cartan system $\left(P_{f}^{*}, C_{f}^{*}\right)$. We can see that $u^{0}$ is reducible on $P_{f}^{*}$. Putting $r=q f$, we get a Cartan system $\left(P_{f}^{1}, \tilde{C}_{f}^{1}\right)$. We can verify that $u_{1}^{0}=2 s-f_{p}$ is an invariant of $\left(P_{f}^{1}\right.$, $\left.\tilde{C}_{f}^{1}\right)$ and that $u_{1}^{0}$ is reducible on $P_{f}^{1}$. Here we use the notations $f_{x}=\partial f / \partial x$, $f_{p}=\partial f / \partial p$ etc. Putting $s=f_{p} / 2$, we get a Cartan system $\left(P_{f}^{2}, \tilde{C}_{f}^{2}\right)$. We can verify that $u_{2}^{0}=t-\left(f_{p p} / 2 q\right)$ is an invariant of $\left(P_{f}^{2}, \tilde{C}_{f}^{2}\right)$ and that $u_{2}^{0}$ is reducible on $P_{f}^{2}$. Putting $t=f_{p p} / 2 q$, we obtain a Cartan system $\left(Q_{f}, C_{f}\right)$, where

$$
Q_{f}=\left\{(x, y, p, q) \in R^{4} ; \quad q \neq 0,(x, y, p) \in U(f)\right\}
$$

and

$$
C_{f}:\left\{\begin{array}{l}
\omega^{1}=d x \\
\omega^{2}=q d y-p q d x \\
\omega^{3}=-q d p+q f \omega^{1}+\left(f_{p} / 2\right) \omega^{2}, \\
\omega^{4}=d q / q+\left(f_{p} / 2\right) \omega^{1}+\left(f_{p p} / 2 q\right) \omega^{2} .
\end{array}\right.
$$

$\left(Q_{f}, C_{f}\right)$ is a defining Cartan system of $\&\left(S_{f}\right)$ (cf. [7] Theorem 2.3). The Pfaffian system to be considered is now given by $S_{f}^{*}=\left\langle\omega^{2}, \omega^{3}\right\rangle$. Let $g$ be another function on an open set of $\boldsymbol{R}^{3}$. Then $S_{f}$ and $S_{g}$ are locally (S)equivalent if and only if $\left(Q_{f}, C_{f}\right)$ and $\left(Q_{g}, C_{g}\right)$ are locally isomorphic (Theorems 1.3 and 2.2.). Therefore we have only to classify the Cartan systems $\left(Q_{f}, C_{f}\right)$. We remark that $\mathbb{S}\left(S_{f}\right)$ is finite dimensional for any $f$.

Now we have the following structure equation of $\left(Q_{f}, C_{f}\right)$ :

Eq. 1)

$$
\left\{\begin{array}{l}
d \omega^{1}=0 \\
d \omega^{2}=\omega^{3} \wedge \omega^{1}+\omega^{4} \wedge \omega^{2}, \\
d \omega^{3}=\omega^{4} \wedge \omega^{3}-u_{3} \omega^{2} \wedge \omega^{1}, \\
d \omega^{4}=u_{1} \omega^{3} \wedge \omega^{2}-u_{2} \omega^{2} \wedge \omega^{1},
\end{array}\right.
$$

where $u_{1}, u_{2}$ and $u_{3}$ are invariants of $\left(Q_{f}, C_{f}\right)$. They determine other invariants $u_{\imath}, 4 \leqq i \leqq 10$, as follows:

Eq. 2)

$$
\left\{\begin{array}{l}
d u_{1}=u_{4} \omega^{1}+u_{5} \omega^{2}+u_{6} \omega^{3}-2 u_{1} \omega^{4}, \\
d u_{2}=u_{7} \omega^{1}+u_{8} \omega^{2}+u_{4} \omega^{3}-u_{2} \omega^{4}, \\
d u_{3}=u_{9} \omega^{1}+u_{10} \omega^{2}-u_{2} \omega^{3} .
\end{array}\right.
$$

We have $u_{1}=-f_{p p p} /\left(2 q^{2}\right)$. If $u_{1}=0$ on $Q_{f}$, then $f, u_{2}$ and $u_{3}$ are given as follows: 
Eq. 3)

$$
\left\{\begin{array}{l}
f=a(x, y) p^{2}+2 b(x, y) p+c(x, y) \\
u_{2}=\left(a_{x}-b_{y}\right) / q \\
u_{3}=\left(a_{x}-b_{y}\right) p+\left(a c-c_{y}+b_{x}-b^{2}\right) .
\end{array}\right.
$$

Using the invariants $u_{1}, u_{2}$ and $u_{3}$, we shall consider four cases. Then we can study each case by using Theorem 1.3.

Before going further, we give the following complete list of classification. We denote by $m$ the dimension of $\leftrightarrow\left(S_{f}\right)$ and by $f_{s}$ the standard form of each type. As usual, we use the notations $F^{\prime}(x)=d F / d x, G_{p}(x, p)$ $=\partial G / \partial p$ etc.
A) $\quad m=4, f_{s}=a y(a \in R)$.
B) $\quad m=3, f_{s}=F(x) y\left(F^{\prime}(x) \neq 0\right)$.
$\left.\mathrm{C}_{1}\right) \quad m=2, f_{s}=(1 / 2 a) p^{2}+2(a+b-y) p+2 a\left(y^{2}-2 b y+(a-b)^{2}\right)$ $(a, b \in R, a \neq 0)$.
$\left.\mathrm{C}_{2}\right) \quad m=2, f_{s}=a p+F(p-a y)\left(a \in R, F^{\prime \prime \prime}(p) \neq 0\right)$.
$\left.\mathrm{D}_{1}\right) \quad m=1, f_{s}=a(x) p^{2}+(F(x)+b(x)-2 a(x) F(x) y) p$

$$
\begin{aligned}
& \quad+a(x) F(x)^{2} y^{2}+\left(F^{\prime}(x)-b(x) F(x)\right) y+c(x) \\
& \left(\begin{array}{l}
a^{\prime}(x)+a(x) F(x)=1, \\
4 a(x) c(x)=(F(x)-b(x))^{2}+2\left(F^{\prime}(x)-b^{\prime}(x)\right)
\end{array}\right) .
\end{aligned}
$$
$\left.\mathrm{D}_{2}\right) \quad m=1, f_{s}=F(x) p+F^{\prime}(x) y+G(x, p-F(x) y)\left(G_{p p p}(x, p) \neq 0\right)$.
$\left.\mathrm{D}_{3}\right) \quad m=1, f_{s}=a(y) p^{2}+2 b p+c(y)\left(b \in R, c^{\prime}(y)=a(y) c(y)-\left(y+b^{2}\right)\right)$.
$\left.\mathrm{D}_{4}\right) \quad m=1, f_{s}=a(y) p^{2}-2 y p+c(y)$.
$\left.\mathrm{D}_{5}\right) \quad m=1, f_{s}=G(y, p)\left(G_{p p p}(y, p) \neq 0\right)$.
$\left.\mathrm{E}_{1}\right) \quad m=0, f_{s}=a(x, y) p^{2}+2 b(x, y) p+c(x, y)$

$$
\left(a_{x}=b_{y}, b_{x}-c_{y}=y+b^{2}-a c, a_{x}^{2}+b_{x}^{2}+c_{x}^{2}>0\right) .
$$
$\left.\mathrm{E}_{2}\right) \quad m=0, f_{s}=a(x, y) p^{2}+2 b(x, y) p+c(x, y)$

$$
\left(a_{x}-b_{y}=1, a_{x}^{2}+b_{x}^{2}+c_{x}^{2}>0\right) .
$$

$\left.\mathrm{E}_{3}\right) \quad m=0, f_{s}=F_{x}(x, y)+F_{y}(x, y) p+G(x, y, p-F(x, y))$

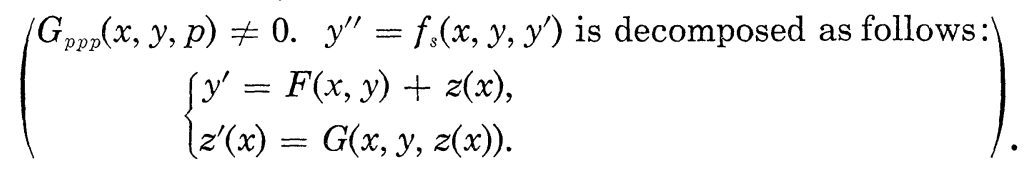

We shall give an outline of the argument.

Case I) $u_{1}, u_{2}$ and $u_{3}$ are constants. Then, from Eq. 2), we have $u_{1}=u_{2}=0$. Putting $a=-u_{3}$, we get the first type A). $\left(\omega^{3}+\sqrt{a} \omega^{2}, \omega^{2}\right)$ is a solvable generator of $S_{f}^{*}$ with auxiliary 1-forms $\omega^{4}-\sqrt{\bar{a}} \omega^{1}, \omega^{4}+\sqrt{\bar{a}} \omega^{1}$.

Case II) $d u_{i}=0, i=1,2$, and $d u_{3} \neq 0$ on $Q_{f}$. Then, $u_{1}=u_{2}=0$ and 
$u_{3}$ is a function of $x$ and $y$.

1) $u_{3}=u_{3}(x)$. Then $u_{3}$ forms a complete system of invariants of $\left(Q_{f}\right.$, $C_{f}$ ). Putting $F(x)=-u_{3}$, we get the second type B). If we know a solution of Riccati's equation $u^{\prime}(x)=u(x)^{2}-F(x)$, then $\left(\omega^{3}-u(x) \omega^{2}, \omega^{2}\right)$ is a solvable generator of $S_{f}^{*}$ with auxiliary 1-forms $\omega^{4}+u(x) \omega^{1}, \omega^{4}-u(x) \omega^{1}$.

2) $\partial u_{3} / \partial y \neq 0$ on $Q_{f}$. Taking an element $X=x, Y=u_{3}(x, y)$ of $\mathbb{S}^{\prime}$, we can assume that $u_{3}=y$. Then, $u_{9}=p, u_{10}=1 / q$ and hence $\operatorname{dim} \mathbb{S}\left(S_{f}\right)$ $\leqq 1$. In this case, $f, a(x, y)$ and $b(x, y)$ are also invariants of $\left(Q_{f}, C_{f}\right)$, where $f$ is given by Eq. 3). From this fact, we get the types $\mathrm{D}_{3}$ ) and $\mathrm{E}_{1}$ ).

Case III) $d u_{1}=0$ and $d u_{2} \neq 0$ on $Q_{f}$. Then, $u_{1}=0$. We can see that $u_{2}$ and $u_{3}$ are reducible on $Q_{f}$. Putting $u_{2}=1$ and $u_{3}=0$, we get a Cartan system $\left(Q_{f}^{1}, C_{f}^{1}\right)$, where $C_{f}^{1}=\left(u_{7}, u_{8}, u_{9}, u_{10} ; \omega^{1}, \omega^{2}\right)$. If all invariants are constants, then we have $u_{7}-u_{10}=1 / u_{8}$ and $u_{9}=u_{10}^{2} / u_{8}$. We get the type $\mathrm{C}_{1}$ ), which depends on two constants. If one of the invariants $u_{7}$, $u_{8}, u_{9}$ and $u_{10}$ is not a constant, then we can proceed as in Case II). If $\operatorname{dim} \circlearrowleft\left(S_{f}\right)=1$, then $\left(Q_{f}^{1}, C_{f}^{1}\right)$ is locally isomorphic to one of the following two cases:

1) $x$ forms a complete system of invariants of $\left(Q_{f}^{1}, C_{f}^{1}\right)$;

2) $y$ forms a complete system of invariants of $\left(Q_{f}^{1}, C_{f}^{1}\right)$.

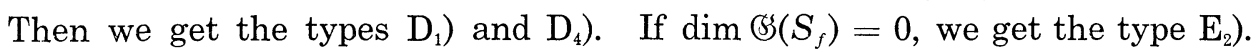

Case IV) $d u_{1} \neq 0$ on $Q_{f}$. We can see that $u_{1}$ is reducible on $Q_{f}$. Putting $u_{1}=1 /(2 a)(a \in R, a \neq 0)$, we get a Cartan system $\left(Q_{f}^{2}, C_{f}^{2}\right)$, where $C_{f}^{2}=\left(u_{2}, u_{3}, u_{4}, u_{5}, u_{6} ; \omega^{1}, \omega^{2}, \omega^{3}\right)$. It is not hard to verify that $\operatorname{dim} \circlearrowleft\left(S_{f}\right) \leqq 2$ and that if $\operatorname{dim} \circlearrowleft\left(S_{f}\right)=2$ then $\left(Q_{f}^{2}, C_{f}^{2}\right)$ does not admit any invariant of the form $u=u(x, y)$. Therefore one of the invariants $u_{i}, 2 \leqq i \leqq 6$, is reducible on $Q_{f}^{2}$. We can carry out the isomorphic reduction and get a Cartan system $\left(Q_{f}^{3}, C_{f}^{3}\right)$, where $C_{f}^{3}=\left(u_{2}, u_{3}, u_{4}, u_{5}, u_{6} ; \omega^{1}, \omega^{2}\right)$. If all invariants of $\left(Q_{f}^{3}, C_{f}^{3}\right)$ are constants, then we get the type $\left.\mathrm{C}_{2}\right)$. If $\operatorname{dim}\left(\mathfrak{S}^{\prime}\left(S_{f}\right)=1\right.$, then an invariant $v(x, y)$ forms a complete system of invariants of $\left(Q_{f}^{3}, C_{f}^{3}\right)$. As in Case II), we can assume that $v=x$ or $v=y$. Then we get the types $\left.\mathrm{D}_{2}\right)$ and $\mathrm{D}_{5}$ ). If $\operatorname{dim} \mathfrak{S}_{(}\left(S_{f}\right)=0$, we get the type $\left.\mathrm{E}_{3}\right)$. Assume that $f$ and $g$ belong to the type $\left.\mathrm{E}_{3}\right)$. Let $\bar{u}_{i}, 2 \leqq i \leqq 6$, denote the invariants of $\left(Q_{g}^{3}\right.$, $C_{g}^{3}$ ). Then the equivalence of $S_{f}$ and $S_{g}$ is determined by the relations $u_{i}=\bar{u}_{i}, 2 \leqq i \leqq 6$. If the relations are compatible, they define a unique element of 'G' which makes $S_{f}$ and $S_{g}$ equivalent. 


\section{REFERENCES}

[1] E. Cartan, Sur l'intégration des systèmes différentiels complètement intégrables, C. R. Acad. Sc., t. 134 (1902), 1415-1418 et 1564-1566.

[2] E. Cartan, Les sous-groupes des groupes continus de transformations, Ann. Ec. Norm., 25 (1908), 57-194.

[ 3 ] E. Cartan, Les systèmes de Pfaff à cinq variables et les équations aux dérivées partielles du second ordre, Ann. Ec. Norm., 27 (1910), 109-192.

[4] E. Cartan, Sur les systèmes en involution d'équations aux dérivées partielles du second ordre à une fonction inconnue de trois variables indépendantes, Oeuvres complètes, Partie II, vol. 2, 1035-1125.

[ 5 ] E. Cartan, Les systèmes différentiels extérieurs et leurs applications géométriques, Hermann, Paris, 1945.

[6 ] E. Cartan, Notice sur les travaux scientifiques, Oeuvres complètes, Partie I, vol. 1, $1-112$.

[ 7 ] T. Higa, On the isomorphic reduction of an invariant associated with a Lie pseudogroup, Comment. Math. Univ. St. Paul., 34 (1985), 163-175.

[ 8 ] H. H. Johnson, Classical differential invariants and applications to partial differential equations, Math. Ann., 148 (1962), 308-329.

[9] A. Kumpera, Invariants différentiels d'un pseudogroupe de Lie, I, J. Differential Geom., 10 (1975), 289-345.

[10] T. Morimoto, Sur le problème d'équivalence des structures géométriques, Japan J. Math., New Series, 9 (1983), 293-372.

[11] I. M. Singer and S. Sternberg, The infinite groups of Lie and Cartan, Part I, J. Analyse Math., 15 (1965), 1-114.

[12] S. Sternberg, Lectures on Differential Geometry, Prentice-Hall, New Jersey, 1964.

Department of Mathematics

Faculty of Science

Rikkyo University

Ikebukuro, Tokyo 171

Japan 